\title{
Rank 3 rigid representations of projective fundamental groups
}

\author{
Adrian Langer, Carlos Simpson
}

\begin{abstract}
Let $X$ be a smooth complex projective variety with basepoint $x$. We prove that every rigid integral irreducible representation $\pi_{1}(X, x) \rightarrow \mathrm{SL}(3, \mathbb{C})$ is of geometric origin, i.e., it comes from some family of smooth projective varieties. This partially generalizes an earlier result by K. Corlette and the second author in the rank 2 case and answers one of their questions.
\end{abstract}

\section{Introduction}

The main examples of local systems in complex algebraic geometry are those that come from families of varieties. Suppose $f: Z \rightarrow X$ is a smooth projective morphism of algebraic varieties. Then the $i$-th higher direct image of the constant sheaf $\mathbb{C}_{Z}$ is a semisimple local system $R^{i} f_{*}\left(\mathbb{C}_{Z}\right)$ on $X$. It furthermore has a structure of polarized $\mathbb{Z}$-variation of Hodge structure with integral structure given by the image of the map $R^{i} f_{*}\left(\mathbb{Z}_{Z}\right) \rightarrow R^{i} f_{*}\left(\mathbb{C}_{Z}\right)$. We will view all the irreducible direct factors of such local systems as coming from geometry.

Somewhat more generally, over a smooth variety $X$ we say that an irreducible $\mathbb{C}$-local system $L$ is of geometric origin if there is a Zariski open dense subset $U \subset X$ and a smooth projective family $f: Z \rightarrow U$ such that $\left.L\right|_{U}$ is a direct factor of $R^{i} f_{*}\left(\mathbb{C}_{Z}\right)$ for some $i$. See Sections 2.5 and 7 for a further discussion of possible variants of this notion.

Local systems of geometric origin are $\mathbb{C}$-variations of Hodge structure. Furthermore, from the $R^{i} f_{*}\left(\mathbb{Z}_{Z}\right)$ they inherit integral structure, in the sense that the traces of monodromy matrices are algebraic integers. Local systems of geometric origin also have a Galois descent property when viewed as $\mathbb{Q}_{\ell}$-local systems over an arithmetic model of $X$, as described in [Si1, Theorem 4].

Several natural questions may be posed. For instance, does an integral local system underlying a $\mathbb{C}$-variation of Hodge structure have geometric origin? 
Recall from Mostow-Margulis rigidity theorems that many local systems naturally occurring over higher dimensional varieties are rigid, i.e. they have no nontrivial deformations. One can easily see that rigid $\mathbb{Q}_{\ell}$-local systems automatically have the Galois descent property mentioned above. Furthermore, it is a consequence of Corlette's theorem that rigid local systems are $\mathbb{C}$-variations of Hodge structure. It is natural to formulate the following conjecture:

CONJECTURE 1.1. Over a smooth projective variety $X$, any rigid local system $L$ is of geometric origin.

This conjecture was proven for local systems on root stacks over $\mathbb{P}^{1}$ by Katz [Ka1], who gives a complete classification and inductive description of rigid local systems in that case. For local systems of rank 2 it was proven by K. Corlette and the second author $[\overline{\mathrm{CS}}]$ who also prove a stronger classification result.

A consequence of the conjecture would be the following subsidiary statement:

CONJECTURE 1.2. Over a smooth projective variety $X$, any rigid local system is integral. 1

In this paper we consider the case of rank 3 , and obtain the following main theorem.

THEOREM 1.3. Let $X$ be a smooth complex projective variety with basepoint $x$. Then every rigid integral irreducible representation $\rho: \pi_{1}(X, x) \rightarrow \operatorname{SL}(3, \mathbb{C})$ is of geometric origin.

Our techniques will not address the question of proving that a local system is integral, so we have included integrality as a hypothesis. Consequently, our theorem does not provide a complete answer to Conjecture 1.1 in the rank 3 case, but it does show for rank 3 that Conjecture 1.2 implies Conjecture 1.1.

On the other hand, for varieties with no symmetric differentials in some range, Conjecture 1.2 was proven by B. Klingler in [K12]. Together with Theorem 1.3 his results imply the following corollary:

COROLlary 1.4. Let $X$ be a smooth projective variety with $H^{0}\left(X, \operatorname{Sym}^{i} \Omega_{X}\right)=0$ for $i=1,2,3$. Then any representation $\pi_{1}(X, x) \rightarrow \mathrm{GL}(3, \mathbb{C})$ is of geometric origin.

We now explain the main ideas of the proof of Theorem 1.3. We say that a variation of Hodge structure (VHS for short) is of weight one if its Hodge types are contained in the set $\{(1,0),(0,1)\}$. It is well known that a polarizable weight one

\footnotetext{
${ }^{1}$ Esnault and Groechenig have recently posted a proof of this conjecture for cohomologically rigid local systems [EG]. See Remark 7.12 for the definition of cohomologically rigid and an application.
} 
$\mathbb{Z}$-VHS comes from an algebraic smooth family of abelian varieties. Therefore, if we can construct such a VHS then we obtain an algebraic family and the underlying local system is of geometric origin. A refined version of this observation, which one can learn for example from Deligne's [Del], goes as follows. Start with a local system $L$ of complex vector spaces, but assume that $L$ is rigid. Then it is defined over some algebraic number field $K \subset \mathbb{C}$. If we also assume that $L$ is integral, then Bass-Serre theory says that there is a local system of projective $\mathscr{O}_{K}$-modules $L_{\mathscr{O}_{K}}$ with

$$
L \cong L_{\mathscr{O}_{K}} \otimes_{\mathscr{O}_{K}} \mathbb{C} \text {. }
$$

The local system $L_{\mathscr{O}_{K}}$ may be viewed as a local system of abelian groups, hence of (free) $\mathbb{Z}$-modules. Now

$$
L_{\mathscr{O}_{K}} \otimes_{\mathbb{Z}} \mathbb{C}=\bigoplus_{\sigma: K \rightarrow \mathbb{C}} L^{\sigma}
$$

where $L^{\sigma}$ is the $\mathbb{C}$-local system obtained by extension of scalars using the embedding $\sigma$. In order to give our $\mathbb{Z}$-local system a structure of polarized weight-one $\mathbb{Z}$-VHS, we should therefore give each complex local system $L^{\sigma}$ a structure of polarized $\mathbb{C}$-VHS of weight one. Adjusting these together in order to have the required properties to get a family of abelian varieties, will be discussed in more detail in Section 7 .

Our original local system $L$ is one of the factors $L^{\sigma_{0}}$ corresponding to the initially given embedding $\sigma_{0}: K \hookrightarrow \mathbb{C}$. The plan to show that $L$ is of geometric origin is therefore to try to put weight-one VHS's on each $L^{\sigma}$. Assuming $L$ is irreducible, so all these local systems are irreducible, if they have structures of VHS then these structures are unique up to translation of Hodge types. The existence of weight-one structures is therefore a property of the $L^{\sigma}$.

In the case $\operatorname{rk}(L)=2$, this property is automatic. Indeed the only two possibilities for the Hodge numbers are a single Hodge number equal to 2, a case we denote (2), or two adjacent Hodge numbers equal to 1, a case we denote $(1,1)$. Notice that if the nonzero Hodge numbers are not adjacent then the Kodaira-Spencer map (Higgs field) must be zero and the system becomes reducible. This explains in a nutshell the procedure that was used in [CS] to treat the rigid and integral local systems of rank 2 .

In the case of local systems of rank 3, there are four possibilities for the type of a VHS: either (3) corresponding to unitary ones, or $(1,2)$ or $(2,1)$ corresponding to weight-one VHS (with Hodge numbers $h^{1,0}=1, h^{0,1}=2$ or $h^{1,0}=2, h^{0,1}=$ 1 respectively); or the last case $(1,1,1)$ when the Hodge bundles are three line bundles. The unitary type (3) can be considered as having weight one in two different ways. Thus we have the following lemma which is implicit in proof of [CS, Theorem 8.1]: 
LemMA 1.5. Suppose $V$ is an irreducible rank 3 local system on a smooth quasiprojective variety $X$. If $V$ is rigid then it underlies either a complex VHS of weight one or a complex VHS of type $(1,1,1)$.

The reasoning up until now has been well-known and standard. The main idea of the present paper is that we can use substantial arguments in birational geometry to rule out the case of VHS type $(1,1,1)$, unless some special behaviour occurs namely factorization through a curve. In turn, the case of factorization through a curve can be shown, again by standard arguments using Katz's theorem, to lead to local systems of geometric origin under the hypothesis of rigidity. We show the following main theorem. See Theorem 5.1 for a more detailed version of part (1) of the conclusion.

THEOREM 1.6. Let $X$ be a smooth complex projective variety with basepoint $x$ and let $\rho: \pi_{1}(X, x) \rightarrow \mathrm{SL}(3, \mathbb{C})$ be an irreducible representation coming from a complex variation of Hodge structure of type $(1,1,1)$. Let $V_{\rho}$ denote the corresponding local system. Then one of the following holds:

1. the image of $\rho$ is not Zariski-dense in $\mathrm{SL}(3, \mathbb{C})$; or

\section{2. $\rho$ projectively factors through an orbicurve.}

Once we have this theorem, the proof of Theorem 1.3 follows the outline described above: the case of factorization through a curve is treated on the side, and otherwise the theorem rules out having a VHS of type $(1,1,1)$. Therefore, all of our local systems $L^{\sigma}$ underly VHS of weight one, and these may be put together into a polarized $\mathbb{Z}$-VHS of weight one corresponding to a family of abelian varieties. The details are described in Section 7 .

We note that Theorem 1.6 generalizes Klingler's result [K11, Proposition 3.3] which had as hypothesis that the Neron-Severi group be of rank 1 .

Let us now explain briefly how we can use arguments from birational geometry to rule out the case of VHS of type $(1,1,1)$. Such a VHS corresponds to a Higgs bundle of the form

$$
E=E^{2,0} \oplus E^{1,1} \oplus E^{0,2}
$$

with $E^{p, q}$ line bundles, and the Higgs field consists of $\theta: E^{2,0} \rightarrow E^{1,1} \otimes \Omega_{X}^{1}$ and $\theta: E^{1,1} \rightarrow E^{0,2} \otimes \Omega_{X}^{1}$. In terms of the VHS $(V, F, \nabla)$, the line bundles are the Hodge bundles $E^{p, q}=F^{p} V / F^{p+1} V$ and the $\theta$ are the Kodaira-Spencer maps induced by $\nabla$. Since the $E^{p, q}$ are line bundles, locally $\theta$ looks like a collection of sections of $\Omega_{X}^{1}$ and the integrability condition $\theta \wedge \theta=0$ implies that these local sections are proportional where nonvanishing. They generate a saturated sub-line bundle $M \subset \Omega_{X}^{1}$. 
For simplicity, let us here assume that $X$ is a surface. Consider the line bundles $L_{1}=E^{2,0} \otimes\left(E^{1,1}\right)^{*}$ and $L_{2}=E^{1,1} \otimes\left(E^{0,2}\right)^{*}$. The sections $\theta$ may be viewed as inclusions $L_{i} \hookrightarrow M \subset \Omega_{X}^{1}$. Write

$$
M=L_{1}\left(B_{1}\right)=L_{2}\left(B_{2}\right)
$$

with $B_{i}$ effective divisors. Bogomolov's lemma (see [Bo, Theorem 4]) says that $M$ cannot be big. We know that the Higgs bundle $(E, \theta)$ is stable, and its rational Chern classes vanish. We are able to conclude several fairly strong properties:

- The line bundles $L_{i}$ both lie on the same line in $N S(X)_{\mathbb{Q}}$;

- At least one of $L_{i}$ is nef of strictly positive degree; and

- $L_{i}^{2}=0$.

That happens in Section 4. The technique is to play off the numerical properties given by stability and vanishing of Chern classes, against the fact that we have effective divisors $B_{i}$ such that $L_{i}+B_{i}$ are contained in $\Omega_{X}^{1}$ and not big by Bogomolov's lemma.

Then in Section 5, assuming for example that $L_{1}$ is nef of strictly positive degree, we write $L_{2}=a \cdot L_{1}$ in the rational Neron-Severi group, with $a \in \mathbb{Q}$, and we try to get information about $a$. Notice that having $L_{1}^{2}=0$ and $L_{1} \hookrightarrow \Omega_{X}^{1}$ allows us to create the rank 2 Higgs bundle $\mathscr{O}_{X} \oplus L_{1}$ corresponding to a projectively flat connection. We would like to view $E$ as being the symmetric square of this rank 2 bundle. In other words, we would like to show that $a=1$ and moreover $L_{1}=L_{2}$.

Part of the difficulty is that there exist VHS's over a curve where $a$ could be somewhat arbitrary, including being negative. Therefore, our strategy is to show that either $a=1$, or else our VHS factors through a map to a curve. In the case $a=1$, again with some further arguments assuming that there is no factorization through a curve, we finally conclude that $L_{1}=L_{2}$ and indeed our VHS was a symmetric square of a rank 2 local system. These arguments use various aspects of the theory of factorization of representations. We include a proof that several different statements of the property "factors through a curve" are equivalent, in the Appendix. The proof of Theorem 1.6 is concluded at the end of Section 5 .

Section 6contains some remarks about the possible extension of this discussion to VHS's of type $(1,1, \ldots, 1)$ in higher ranks, and Section 6 derives the corollaries about local systems of geometric origin leading to the proof of Theorem 1.3 .

\subsection{Notation}

Let us recall that a Higgs bundle is a pair $(E, \theta)$ consisting of a locally free $\mathscr{O}_{X^{-}}$ module and an $\mathscr{O}_{X}$-linear map $\theta: E \rightarrow E \otimes \Omega_{X}$ such that $\theta \wedge \theta=0$. A system of 
Hodge bundles is a Higgs bundle $(E, \theta)$ with a decomposition $E=\bigoplus E^{p, q}$ such that $\theta$ maps $E^{p, q}$ into $E^{p-1, q+1} \otimes \Omega_{X}$. We say that a system of Hodge bundles $(E, \theta)$ is of type $\left(i_{0}, \ldots, i_{n}\right)$ if

$$
\operatorname{rk} E^{p, q}= \begin{cases}i_{q} & \text { if } p+q=n \\ 0 & \text { if } p+q \neq n\end{cases}
$$

and all the maps $\theta: E^{m-q, q} \rightarrow E^{m-q-1, q+1} \otimes \Omega_{X}$ are non-zero for $q=0, \ldots, n-$ 1. Similarly, replacing $\Omega_{X}$ with $\Omega_{X}(\log D)$ one can define logarithmic systems of Hodge bundles.

In the following we make the convention that complex variation of Hodge structure (VHS) always means polarizable VHS. Giving an irreducible VHS on a smooth complex projective variety is equivalent to giving a stable system of Hodge bundles with vanishing rational Chern classes.

We say that a complex VHS is of type $\left(i_{0}, \ldots, i_{n}\right)$ if the corresponding system of Hodge bundles is of type $\left(i_{0}, \ldots, i_{n}\right)$.

Let $X$ be a smooth complex projective variety. Let us recall that a line bundle is called nef if it has a non-negative degree on every irreducible projective curve in $X$. Let us recall that after Miles Reid, "nef" stands for "numerically eventually free". A line bundle $L$ is called big if its Iitaka's dimension $\kappa(L)$ is equal to the dimension of $X$.

A $\mathbb{Q}$-divisor $D$ is called effective if it can be written as $D=\sum a_{i} D_{i}$, where $D_{i}$ are prime divisors and $a_{i}$ are non-negative rational numbers. We write $D_{1} \geq D_{2}$ for two $\mathbb{Q}$-divisors $D_{1}, D_{2}$, if their difference $\left(D_{1}-D_{2}\right)$ is an effective $\mathbb{Q}$-divisor.

If $L_{1}$ and $L_{2}$ are line bundles then we write $L_{1} \geq L_{2}$ if $H^{0}\left(L_{1} \otimes L_{2}^{-1}\right) \neq 0$.

For various other definitions and properties of line bundles we refer the reader to [Laz, Theorem 2.2.16]. Let us just mention that if $L$ is nef then it is big if and only if $L^{\operatorname{dim} X}>0$.

A morphism $f: X \rightarrow Y$ between smooth quasi-projective varieties (or just orbifolds) is called a fibration if it is surjective and the fibers of $f$ are connected.

A morphism $f: X \rightarrow Y$ is called an alteration if $X$ is smooth and $f$ is proper, surjective and generically finite.

In the following we often abuse notation and we do not distinguish between an algebraic variety and the underlying analytic space. For example, the fundamental group $\pi_{1}(X)$ and cohomology groups $H^{*}(X, \mathbb{Q})$ of an algebraic variety $X$ always denote the corresponding notion for the underlying analytic space. Similarly, essentially all varieties, bundles and maps are algebraic. In the projective case this convention is harmless due to GAGA type theorems but in general this could lead 
to some confusion so in case we use non-algebraic structures we explicitly say so (see the use of analytic maps in the proof of Theorem 5.1).

Let $X$ be a smooth complex quasi-projective variety. Let us fix a smooth projective compactification $\bar{X}$ of $X$ such that $D=\bar{X}-X$ is a simple normal crossing divisor. Let $\left\{\gamma_{i}\right\}$ be loops going around the irreducible components of $D$. Let $\rho: \pi_{1}(X, x) \rightarrow G$ be a representation of $\pi_{1}(X, x)$ in some reductive group $G$ and let us assume that all $\rho\left(\gamma_{i}\right)$ are quasiunipotent. Let us denote by $C_{i}$ the closure of the conjugacy class of $\rho\left(\gamma_{i}\right)$. We say that $\rho: \pi_{1}(X, x) \rightarrow G$ is rigid if it represents an isolated point in the moduli space $M\left(X, G,\left\{C_{i}\right\}\right)$ of representations of $\tau: \pi_{1}(X, x) \rightarrow G$ with conjugacy classes of $\tau\left(\gamma_{i}\right)$ contained in $C_{i}$.

\section{Preliminaries}

\subsection{Homotopy exact sequence}

Let $C$ be a smooth complex quasi-projective curve and let $X$ be a smooth complex quasi-projective variety. Let $f: X \rightarrow C$ be a fibration and let $c$ be a closed point of $C$. Let us recall that the multiplicity of a fiber $F=\sum a_{i} F_{i}$, where $F_{i}$ are irreducible components of $F$, is equal to the greatest common divisor of the coefficients $a_{i}$. Let $p_{1}, \ldots, p_{k}$ be all the points of $C$ at which the fiber of $f$ is multiple (i.e., it has multiplicity at least 2) and let $m_{j}$ denote the multiplicity of the fiber of $f$ over $p_{j}$. Let $c$ be a point of $C$ over which the fiber of $f$ has multiplicity 1 .

We define the orbifold fundamental group $\pi_{1}^{\text {orb }}\left(C_{f}, c\right)$ of $C$ with respect to $f$ as the quotient of $\pi_{1}\left(C-\left\{p_{1}, \ldots, p_{k}\right\}, c\right)$ by the normal subgroup generated by all the elements of the form $\gamma_{j}^{m_{j}}$, where $\gamma_{j}$ is a simple loop going around the point $p_{j}$.

The following theorem is well-known:

THEOREM 2.1. Let $c \in C-\left\{p_{1}, \ldots, p_{k}\right\}$ and let $x$ be a closed point of the fibre $X_{c}$ of $f$ over $c$. Let us assume that either $f$ is proper or $c \in C$ is general. Then the following sequence of groups is exact:

$$
\pi_{1}\left(X_{c}, x\right) \rightarrow \pi_{1}(X, x) \rightarrow \pi_{1}^{\text {orb }}\left(C_{f}, c\right) \rightarrow 1 .
$$

In particular, if we also assume that $f$ has no multiple fibers then the sequence

$$
\pi_{1}\left(X_{c}, x\right) \rightarrow \pi_{1}(X, x) \rightarrow \pi_{1}(C, c) \rightarrow 1
$$

is exact.

Proof. If all the fibers of $f$ have at least one smooth point then the theorem is due to M. Nori [No, Lemma 1.5] (for general fibre) with a small improvement due to O. 
Debarre [De, Lemma 8.11] allowing to deal with arbitrary fibers in the proper case. Nori's proof with appropriate changes as in [Xi] works also for general fibers even without assumption that all the fibres have at least one smooth point. In the general proper case, the assertion comes from [Xi, Lemma 1, Lemma 2 and Lemma 3] in the surface case, but Xiao's proof also works in the general case. Alternatively, for smooth fibers the general case can be reduced to the surface case by the Lefschetz hyperplane theorem. Namely, if $\operatorname{dim} X \geq 3$ then by Bertini's theorem we can find a very ample divisor $H$ such that both $X_{c} \cap H$ and $H$ are smooth and connected. Since, by the Lefschetz hyperplane theorem, the map $\pi_{1}\left(X_{c} \cap H\right) \rightarrow \pi_{1}\left(X_{c}\right)$ is surjective and the map $\pi_{1}(H) \rightarrow \pi_{1}(X)$ is an isomorphism, exactness of the homotopy sequence for $f$ follows from exactness of the homotopy sequence for $\left.f\right|_{H}: H \rightarrow C$. Now the required assertion follows from the surface case by induction.

Remark 2.2. If $f$ is not proper then the homotopy sequence from the above theorem need not be exact even if we assume that all the fibers of $f$ are smooth. One can easily construct examples when this sequence is not exact by blowing up a smooth surface fibered over a curve and removing non-exceptional components in the fiber over the blown-up point.

\subsection{Intersection pairing}

Let $X$ be a smooth complex projective variety of dimension $d \geq 2$. Let $A$ be a fixed very ample divisor on $X$. Then we use intersection pairing on $\mathbb{Q}$-divisors given by

$$
D_{1} \cdot D_{2}:=D_{1} \cdot D_{2} \cdot A^{d-2} \text {. }
$$

We will often use the fact that if $D . A=D^{2}=0$ then the class of $D$ in $H^{2}(X, \mathbb{Q})$ is 0 . To prove that note that if $Y \in|A| \cap \ldots \cap|A|$ denotes a general complete intersection surface in $X$ then the class of $\left.D\right|_{Y}$ in $H^{2}(Y, \mathbb{Q})$ is zero by the Hodge index theorem. But by Lefschetz' hyperplane theorem the restriction $H^{2}(X, \mathbb{Q}) \rightarrow H^{2}(Y, \mathbb{Q})$ is injective so the class of $D$ in $H^{2}(X, \mathbb{Q})$ is also zero.

In the following we write $D_{1} \equiv D_{2}$, if for every $\mathbb{Q}$-divisor $D$ we have $D_{1} . D=$ $D_{2}$.D. In that case Lefschetz' hyperplane theorem implies that the class of $\left(D_{1}-\right.$ $\left.D_{2}\right)$ in $H^{2}(X, \mathbb{Q})$ is equal to zero. $L_{1} \equiv L_{2}$ for line bundles $L_{1}$ and $L_{2}$ denotes equality $c_{1}\left(L_{1}\right) \equiv c_{1}\left(L_{2}\right)$ in $H^{2}(X, \mathbb{Q})$. In that case $L_{1} \otimes L_{2}^{-1} \in \operatorname{Pic}^{\tau} X$.

If $\operatorname{dim} X=2$ then an effective $\mathbb{Q}$-divisor $N=\sum a_{i} N_{i}$, where $a_{i}>0$ and $N_{i}$ are prime, is called negative if the intersection matrix $\left[N_{i} \cdot N_{j}\right]$ is negative definite. It is called semi-negative if the matrix $\left[N_{i} \cdot N_{j}\right]$ is negative semi-definite. By convention, the zero divisor is both negative and semi-negative. 


\subsection{Zariski semi-decomposition}

Let $X$ be a smooth complex projective surface. We say that the degree of a $\mathbb{Q}$ divisor $L$ on $X$ is strictly positive if for every ample divisor $H$ we have $L . H>0$.

Lemma 2.3. Let L be a $\mathbb{Q}$-divisor on $X$. Assume that L.A $>0$ for some ample $A$ and $L^{2} \geq 0$. If $L$ is not big then $L$ is nef, $L^{2}=0$ and the degree of $L$ is strictly positive.

Proof. Let us first note that $L$ is pseudoeffective, i.e., its intersection with any nef divisor is non-negative. Indeed, if $L . H<0$ for some nef $H$ then there exists a positive rational number $a$ such that $L .(A+a H)=0$. Since $A+a H$ is ample, by the Hodge index theorem we get $L^{2}<0$, a contradiction.

Now recall that pseudoeffective divisors admit the so called Zariski decomposition. This means that $L$ can be written as a sum $P+N$, where $P$ is a nef $\mathbb{Q}$-divisor and $N$ is a negative effective $\mathbb{Q}$-divisor with $P . N=0$. Since $L$ is not big, $P$ is also not big which implies that $P^{2}=0$. But in this case if $N \neq 0$ then $L^{2}=N^{2}<0$, a contradiction. Therefore $L=P$ so $L$ is nef with $L^{2}=0$.

To prove that the degree of $L$ is strictly positive it is sufficient to show that there are no ample divisors $H$ with $L . H=0$. But if such a divisor $H$ exists then by the Hodge index theorem $L$ is numerically trivial contradicting inequality $L . A>0$.

Lemma 2.4. Let $C$ be a $\mathbb{Q}$-divisor on $X$. Assume that $C=L+B$, where $L$ is nef and $B$ is effective. If $C$ is not big then the following conditions are satisfied:

1. $L^{2}=0$,

2. B is semi-negative,

3. $L . B=0$. In particular, if we write $B=\sum a_{i} C_{i}$, where $a_{i}>0$ and $C_{i}$ are prime divisors, then $L . C_{i}=0$ for every $i$.

In this case we say that $C=L+B$ is a Zariski semi-decomposition of $C$.

Note that unlike the usual Zariski decomposition, Zariski semi-decomposition need not be unique, i.e., one divisor can have many different Zariski semi-decompositions.

Proof. Since $L$ is nef we have $L^{2} \geq 0$. If $L^{2}>0$ then $L$ is big. But then $C$ is big contradicting our assumption. This proves the first assertion.

To prove the second one, it is sufficient to take any combination $\sum \alpha_{i} C_{i}$ with rational numbers $\alpha_{i} \in\left[0, a_{i}\right]$ and prove that $\left(\sum \alpha_{i} C_{i}\right)^{2} \leq 0$. 
Let us take a rational number $\beta \in[0,1]$. Since $\left(\beta L+\sum \alpha_{i} C_{i}\right) \leq C$ and $C$ is not big, the $\mathbb{Q}$-divisor $\left(\beta L+\sum \alpha_{i} C_{i}\right)$ is not big. But $\left(\beta L+\sum \alpha_{i} C_{i}\right) . A \geq 0$, so we have $\left(\beta L+\sum \alpha_{i} C_{i}\right)^{2} \leq 0$. Putting $\beta=0$, we get the required inequality.

Putting $\beta=1$ we see that for any $\varepsilon \in[0,1]$, we have $(L+\varepsilon B)^{2} \leq 0$. Since $L^{2}=0$, this gives $2 L . B+\varepsilon B^{2} \leq 0$. Passing with $\varepsilon$ to 0 we get $L . B \leq 0$. But $L$ is nef and $B$ is effective so we get $L . B=0$.

\subsection{Lifting of representations of the fundamental group}

Let $G / \mathbb{C}$ be a connected, semisimple group and let $\Gamma$ be a group. A homomorphism of groups $\rho: \Gamma \rightarrow G$ is called irreducible if the Zariski closure of the image of $\Gamma$ is not contained in any proper parabolic subgroup of $G$. In case $G=\operatorname{SL}(n, \mathbb{C})$, this notion is equivalent to the usual notion of an irreducible representation.

Let us recall the following definition (cf. [d], 2.14]):

Definition 2.5. A homomorphism $\rho: \Gamma \rightarrow G$ is called Lie irreducible if it is irreducible and remains so on all finite index subgroups of $\Gamma$.

Let $\rho: \Gamma \rightarrow G$ be an irreducible homomorphism and let $G_{\rho} \subset G$ be the Zariski closure of the image of $\rho$. Then $\rho$ is Lie irreducible if and only if the inclusion of the connected component $G_{\rho}^{0}$ of $G_{\rho}$ into $G$ is irreducible. In that case $G_{\rho}$ is semisimple and its centralizer in $G$ is finite.

The following lemma is a stronger version of [Co, Theorem 3.1]:

LEMMA 2.6. Let $X$ be a smooth, complex, quasi-projective variety and let $\rho$ : $\pi_{1}(X, x) \rightarrow G$ be a homomorphism into a complex, linear algebraic group $G$ and let $\tilde{G} \rightarrow G$ be a central isogeny. Then there exists a finite surjective morphism $p: Z \rightarrow X$ such that $p^{*} \rho: \pi_{1}(Z, z) \rightarrow G$ lifts to $\pi_{1}(Z, z) \rightarrow \tilde{G}$. Moreover, for any completion $X \subset \bar{X}$ there exists an alteration $\bar{p}: \bar{Z} \rightarrow \bar{X}$, such that $\bar{Z}$ is smooth and projective, $D=\bar{Z}-Z$ is a simple normal crossing divisor and the restriction $p=\left.\bar{p}\right|_{p^{-1} X}: Z=p^{-1}(X) \rightarrow X$ is such that $p^{*} \rho: \pi_{1}(Z, z) \rightarrow G$ can be lifted to $\pi_{1}(Z, z) \rightarrow \tilde{G}$

Proof. Let $A$ be the finite abelian group defined by the short exact sequence

$$
1 \rightarrow A \rightarrow \tilde{G} \rightarrow G \rightarrow 1 \text {. }
$$

Since $A$ is contained in the center of $\tilde{G}$, this sequence induces an exact sequence

$$
H^{1}(X, \tilde{G}) \rightarrow H^{1}(X, G) \stackrel{\delta}{\rightarrow} H^{2}(X, A) .
$$

By the comparison theorem, for a finite abelian group $A$ we have a natural isomorphism $H^{2}(X, A) \simeq H_{e ̂ t}^{2}(X, A)$ of the complex cohomology group with an étale 
cohomology group. By [ $[\mathrm{Bh}$, Theorem 1.1] there exists a finite surjective morphism $p: Z \rightarrow X$ such that the image of the obstruction class $\delta([\rho])$ in $H_{e t t}^{2}(Z, A)$ vanishes. This shows that $p^{*} \rho: \pi_{1}(Z, z) \rightarrow G$ can be lifted to a homomorphism $\rho_{Z}: \pi_{1}(Z, z) \rightarrow \tilde{G}$.

The second part of the lemma follows from the first one and from existence of $\log$ resolution of singularities of the normalization of $\bar{X}$ in the function field of $Z$.

Let us recall that a linear algebraic group is called almost-simple, if it does not contain any Zariski closed, connected normal subgroups of positive dimension. Clearly, an almost-simple group is semi-simple and connected.

Proposition 2.7. Let $X$ be a smooth complex quasi-projective variety and let $G / \mathbb{C}$ be a connected, semisimple group. Let $\rho: \pi_{1}(X, x) \rightarrow G$ be an irreducible homomorphism. Then one of the following holds:

1. There exists a finite étale covering $p: Z \rightarrow X$ such that $p^{*} \rho: \pi_{1}(Z, z) \rightarrow G$ is not irreducible.

2. There exists a finite étale covering $p: Z \rightarrow X$ such that the Zariski closure of the image of $p^{*} \rho$ in $G$ is almost-simple and irreducible.

3. There exists a finite surjective morphism $p: Z \rightarrow X$ and simple, simply connected groups $H_{1}, \ldots, H_{m}, m \geq 2$, such that $p^{*} \rho: \pi_{1}(Z, z) \rightarrow G$ factors through a homomorphism $\tau: \pi_{1}(Z, z) \rightarrow H_{1} \times \ldots \times H_{m}$ with a Zariski dense image.

Proof. There exists a finite étale covering $Z \rightarrow X$ such that $\pi_{1}(Z, z)$ maps into the connected component $H=G_{\rho}^{0}$ of the Zariski closure of the image of $\pi_{1}(Z, z)$ in $G$. If $H \subset G$ is not irreducible then we are in the first case. So let us assume that $H \subset G$ is irreducible. Then $H$ has a canonical decomposition into an almost direct product of its almost-simple factors (see [Bor, Theorem 22.10]). More precisely, let $H_{1}, \ldots, H_{m}$ be the minimal elements among the closed, connected normal subgroups of $H$ of positive dimension. Then all $H_{i}$ are almost-simple and the isogeny $H_{1} \times$ $\ldots \times H_{m} \rightarrow H$ is central (with finite kernel). If $m=1$ then we are in the second case. If $m \geq 2$ then we can pass to the universal covering of the product $H_{1} \times \ldots \times H_{m}$ and we are in the last case by Lemma 2.6 .

Remark 2.8. The second case of the above proposition is particularly interesting in presence of fibrations. More precisely, if $f: X \rightarrow Y$ is a fibration, $G$ is almostsimple and $\rho: \pi_{1}(X, x) \rightarrow G$ has Zariski dense image, then the image of the restriction of $\rho$ to a smooth fiber of $f$ is either Zariski dense in $G$ or it is finite (see 
[Zu, Proposition 2.2.2]). In the second case, after some blowing up and taking a finite étale cover, one can factor $\rho$ through the induced fibration (see [Zu, Lemma 2.2.3]).

\subsection{Representations of geometric origin}

Let $X$ be a smooth complex quasiprojective variety and let $\rho: \pi_{1}(X, x) \rightarrow \operatorname{SL}(n, \mathbb{C})$ be a semisimple representation. Let us recall the following definition from the Introduction:

Definition 2.9. We say that a semisimple representation $\rho$ and its associated local system $V$ are of geometric origin if there exists a dense Zariski open subset $U \subset X$ and a smooth projective morphism $f: Z \rightarrow U$ such that $\left.V\right|_{U}$ occurs as a subquotient of the local system $R^{i} f_{*}\left(\mathbb{C}_{Z}\right)$ for some $i$.

Proposition 2.10. If $V_{1}$ and $V_{2}$ are local systems then $V_{1} \oplus V_{2}$ is of geometric origin if and only if $V_{1}$ and $V_{2}$ are. If $V_{1}$ and $V_{2}$ are of geometric origin then so is $V_{1} \otimes V_{2}$. A rank 1 local system is of geometric origin if and only if it is of finite order.

If $p: Y \rightarrow X$ is a generically surjective map between irreducible varieties, and $V$ is a semisimple local system on $X$, then $V$ is of geometric origin if and only if $p^{*}(V)$ is of geometric origin.

Suppose that $\rho_{1}$ and $\rho_{2}$ are two irreducible representations with determinants of finite order, whose projectivizations are isomorphic. Then $\rho_{1}$ is of geometric origin if and only if $\rho_{2}$ is.

Proof. Given families for $V_{1}$ and $V_{2}$ we can take a disjoint union of products with fixed varieties for $V_{1} \oplus V_{2}$. One may also stay within the world of fibrations with connected fibers by then embedding the resulting family in a big projective space and blowing up along that locus. If $V_{1} \oplus V_{2}$ is of geometric origin then $V_{1}$ and $V_{2}$ are so by definition. If $V_{1}$ and $V_{2}$ are of geometric origin, taking the fiber product of the families results, by the Künneth formula, in a family for $V_{1} \otimes V_{2}$.

A rank 1 system of finite order is a direct factor in the 0-th direct image of a finite ramified cover. In the other direction, let $V$ be a rank 1 local system of geometric origin. Let $f: Z \rightarrow U \subset X$ be the corresponding map. Note that $R^{i} f_{*} \mathbb{C}_{Z}$ comes with an integral structure given by the image of $R^{i} f_{*} \mathbb{Z}_{Z} \rightarrow R^{i} f_{*} \mathbb{C}_{Z}$. Hence the eigenvalues of all monodromy transformations are algebraic integers, so any direct summand of $R^{i} f_{*} \mathbb{C}_{Z}$ is integral. The action of $\mathrm{Gal}(\mathbb{C} / \mathbb{Q})$ takes one direct summand to another. Now existence of a polarization implies that any rank 1 direct summand is unitary. Thus, $V$ and all its $\operatorname{Gal}(\mathbb{C} / \mathbb{Q})$-conjugates are integral and unitary. But by Kronecker's theorem, an algebraic integer all of whose conjugates 
have absolute value 1 is a root of unity, so $V$ is of finite order (not only on $U$ but also on $X$ ).

Suppose $p: Y \rightarrow X$ is a generically surjective map and $V$ a local system on $X$. If $V$ is of geometric origin on $X$ then since the image of $p$ intersects the open set $U \subset X$ on which $V$ is defined, we get a family over $p^{-1}(U)$ showing that $p^{*}(V)$ is of geometric origin.

In the other direction, suppose $p^{*}(V)$ is of geometric origin coming from a family $f: Z \rightarrow U$ with $U \subset Y$. Choose a subvariety $Y^{\prime} \subset U$ which is quasi-finite over $X$. By replacing $Y^{\prime}$ by a Zariski open subset, we may assume that $p^{\prime}: Y^{\prime} \rightarrow U^{\prime}$ is a finite étale map to a dense open subset $U^{\prime} \subset X$. Let $Z^{\prime} \subset Z$ be the inverse image of $Y^{\prime}$, and denote by $f^{\prime}: Z^{\prime} \rightarrow Y^{\prime}$ the map. Under our suppositions, it is smooth and projective, hence also the composed map

$$
Z^{\prime} \stackrel{f^{\prime}}{\rightarrow} Y^{\prime} \stackrel{p^{\prime}}{\rightarrow} U^{\prime}
$$

is smooth and projective. By the decomposition theorem, $R^{i}\left(f^{\prime}\right)_{*}\left(\mathbb{C}_{Z^{\prime}}\right)$ is semisimple so a subquotient may be viewed as a direct factor. Thus, we may assume given an injective map $\left(p^{\prime}\right)^{*}(V) \rightarrow R^{i}\left(f^{\prime}\right)_{*}\left(\mathbb{C}_{Z^{\prime}}\right)$ which gives by composition, in turn, an injective map

$$
\left.V\right|_{U^{\prime}} \rightarrow\left(p^{\prime}\right)_{*}\left(p^{\prime}\right)^{*}(V) \rightarrow R^{i}\left(p^{\prime} f^{\prime}\right)_{*}\left(\mathbb{C}_{Z^{\prime}}\right),
$$

showing that $V$ is of geometric origin.

For the last part, suppose given two irreducible representations $\rho_{1}$ and $\rho_{2}$ whose determinants are of finite order. Let $V_{1}$ and $V_{2}$ respectively denote the corresponding local systems. Suppose the projectivizations of $\rho_{1}$ and $\rho_{2}$ are isomorphic, and fix bases for $\left(V_{1}\right)_{x}$ and $\left(V_{2}\right)_{x}$ at the basepoint $x \in X$ that are compatible with the isomorphism of projectivizations. For any $\gamma \in \pi_{1}(X, x)$ we may then write

$$
\rho_{2}(\gamma)=a(\gamma) \rho_{1}(\gamma)
$$

with $a(\gamma)$ a diagonal matrix. Now $a: \pi_{1}(X, x) \rightarrow \mathbb{C}^{*}$ is a rank 1 representation corresponding to a rank 1 local system $A$, and we have $V_{2}=A \otimes V_{1}$. If $r=\operatorname{rk}\left(V_{1}\right)$ then

$$
\operatorname{det}\left(V_{2}\right)=A^{\otimes r} \otimes \operatorname{det}\left(V_{1}\right) .
$$

From the hypothesis, $A^{\otimes r}$ is of finite order, so $A$ is of finite order. Hence, $A$ is of geometric origin. It follows that $V_{2}$ is of geometric origin if and only if $V_{1}$ is.

Concerning the second paragraph, it is also true that any pullback of a system of geometric origin, is of geometric origin. For the case of pullback along a map that goes into the complement of the open set $U$ over which the smooth family is given, 
this requires using the theory of perverse sheaves and applying the decomposition theorem; we do not treat that here.

The converse to the part about $V_{1} \otimes V_{2}$ looks like an interesting question: if $V_{1}$ and $V_{2}$ are irreducible local systems with trivial determinant such that $V_{1} \otimes V_{2}$ is of geometric origin then are $V_{1}$ and $V_{2}$ of geometric origin?

Our definition of local systems of geometric origin is rather large, and one can envision a couple of strengthenings. The following remarks on this point are not needed in the rest of the paper: we refer everywhere only to the notion of Definition 2.9, and leave open the question of obtaining the stronger properties we now mention.

First, one could ask for $U=X$, that is to say one could ask for a smooth family extending over $X$. We might say in that case that $V$ is smoothly of geometric origin. This looks to be a very strong condition, and it is difficult to predict when it should be satisfied. There are natural examples of local systems coming from families of varieties, such that the local system extends to a larger subset than the subset of definition of the smooth family. Indeed, any even-dimensional Lefschetz pencil has order two monodromy around the singularities, so after pulling back to a twofold cover of the base ramified at the singular points, the local system extends. It is highly unclear whether to expect the existence of a smooth globally defined family realising the same monodromy representation.

In particular, Katz's theorem that rigid local systems over the orbifold projective line are of geometric origin, does not give smooth geometric origin. The discussion of the notion of geometric origin in [To2] should probably be modified accordingly.

Another point is the question of whether the projector to our direct factor is motivic. Say that a local system $V$ is strongly of geometric origin if there exists a proper morphism $f: Z \rightarrow X$ and an algebraic cycle representing a projector $\pi$ : $R f_{*}\left(\mathbb{Z}_{Z}\right) \rightarrow R f_{*}\left(\mathbb{Z}_{Z}\right)$ such that $V$ is the image of $\pi$.

One would need at least the Hodge conjecture to go from our (weak) notion of geometric origin to this notion of strong geometric origin, but in fact it does not even seem clear that the Hodge conjecture would be sufficient for that.

For instance one would like to answer the following question (a punctual analogue of the converse question for tensor products of local systems mentioned above): given $\mathbb{Z}$-Hodge structures $V_{1}$ and $V_{2}$ with trivial determinants such that $V_{1} \otimes V_{2}$ is a motive, are $V_{1}$ and $V_{2}$ motives?

If we do not know the answer to that question, but do assume the Hodge conjecture, then one might be able to show that $V$ of weak geometric origin implies that some $V^{\oplus k}$ is of strong geometric origin. Some understanding of algebraic cycles and the decomposition theorem would also clearly be needed. 
In the context of the present paper, our main constructions provide smooth families of abelian varieties, hence give smooth geometric origin in some cases. One can envision looking at the question of strong geometric origin for those cases - that would require delving into the endomorphism algebras of the families of abelian varieties we construct. We do not make any claims about that here.

\section{Factorization through orbicurves}

An orbicurve $C$ is a smooth 1-dimensional Deligne-Mumford stack whose generic stabilizer group is trivial. The coarse moduli space $C^{\text {coarse }}$ is a smooth curve having a collection of points $p_{1}, \ldots, p_{k}$ such that there exist strictly positive integers $n_{1}, \ldots, n_{k}$ such that

$$
C \cong C^{\text {coarse }}\left[\frac{p_{1}}{n_{1}}, \ldots, \frac{p_{k}}{n_{k}}\right]
$$

is the root stack. The fundamental group $\pi_{1}(C, y)$ is the same as the orbifold fundamental group considered in Subsection 2.1 (with $n_{i}$ equal to multiplicities of the fibres over $p_{i}$ ).

The goal of this subsection is to prove the equivalence of several different notions of factorization through orbicurves.

Definition 3.1. Let $X$ be a smooth complex quasi-projective variety. Suppose that $\rho: \pi_{1}(X, x) \rightarrow \mathrm{GL}(n, \mathbb{C})$ is an irreducible representation.

1. We say that $\rho$ projectively factors through an orbicurve if there is an orbicurve $C$, a fibration $f: X \rightarrow C$, and a commutative diagram

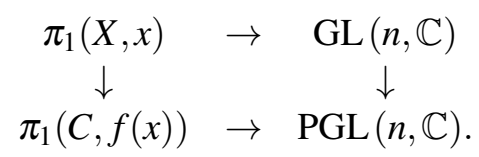

2. We say that $\rho$ virtually projectively factors through an orbicurve if there is an alteration $p: Z \rightarrow X$ such that $p^{*} \rho$ projectively factors through an orbicurve.

Definition 3.2. Let $X$ be a smooth complex quasi-projective variety. We say that an irreducible representation $\rho: \pi_{1}(X, x) \rightarrow \operatorname{GL}(n, \mathbb{C})$ is tensor decomposable if the associated local system $V_{\rho}$ can be written as a tensor product $V_{1} \otimes V_{2}$ of two local systems of rank $\geq 2$. Say that $\rho$ is virtually tensor decomposable if there exists an alteration $p: Z \rightarrow X$ such that $p^{*} \rho$ is tensor decomposable.

Say that $\rho$ is virtually reducible if there is an alteration $p: Z \rightarrow X$ such that $p^{*} \rho$ is reducible, i.e. it decomposes into a nontrivial direct sum. 
Let us note that a representation $\rho: \pi_{1}(X, x) \rightarrow \operatorname{SL}(n, \mathbb{C})$ is virtually reducible if and only if it is not Lie irreducible. Indeed, if $p: Z \rightarrow X$ is an alteration then the image of $\pi_{1}(Z, z) \rightarrow \pi_{1}(X, x)$ has finite index in $\pi_{1}(X, x)$. So if $\rho$ is virtually reducible then it is not Lie irreducible. The implication in the other direction follows from the fact that a finite index subgroup in $\pi_{1}(X, x)$ gives rise to a finite étale covering.

For the proof of the following factorization theorem we refer to Appendix (see Theorem 8.4):

THEOREM 3.3. Let $X$ be a smooth complex projective variety. Let us fix an irreducible representation $\rho: \pi_{1}(X, x) \rightarrow \operatorname{SL}(n, \mathbb{C})$ for some $n \geq 2$. Suppose that $\rho$ is not virtually tensor decomposable, and not virtually reducible. Then the following conditions are equivalent.

1. $\rho$ projectively factors through an orbicurve;

2. $\rho$ virtually projectively factors through an orbicurve;

3. There exists a map $f: X \rightarrow C$ to an orbicurve and a fiber $F=f^{-1}(y)$, such that the restriction of $\rho$ to $\pi_{1}(F, x)$ becomes reducible;

4. There exists an alteration $p: Z \rightarrow X$ such that the previous condition holds for the pullback $p^{*} \rho$.

Lemma 3.4. Suppose $\rho: \pi_{1}(X, x) \rightarrow \operatorname{SL}(n, \mathbb{C})$ is an irreducible representation of rank $n \leq 3$. Then $\rho$ is not virtually tensor decomposable. If $\rho$ is virtually reducible then it has image either in a finite subgroup, or in the normalizer of a maximal torus. If $\rho$ is virtually reducible and underlies a VHS then it is unitary with a single Hodge type.

Proof. Clearly $\rho$ cannot be virtually tensor decomposable because then it should have rank $\geq 4$. Suppose $\rho$ is virtually reducible, becoming reducible upon pullback to $p: Z \rightarrow X$. The image of $\pi_{1}(Z, z)$ is of finite index in $\pi_{1}(X, x)$ and the restriction of $\rho$ to this subgroup is reducible. Therefore we may replace $Z$ by the finite étale covering corresponding to this subgroup, in other words we may suppose that $p$ is finite étale. We may furthermore suppose it is Galois with group $G$. Write the decomposition into isotypical components

$$
\left.V_{\rho}\right|_{Z}=\bigoplus V_{i} \otimes W_{i}
$$

where $V_{i}$ are irreducible local systems on $Z$ and $W_{i}$ are vector spaces. The Galois group acts on $\left\{V_{i}\right\}$. This action is transitive, otherwise $\rho$ would be reducible. In particular, all of the $V_{i}$ and all of the $W_{i}$ have the same rank. 
Suppose there is more than one isotypical component. Then $V_{i}$ and $W_{i}$ must be of rank 1 and $G$ acts on the set of isotypical components by permutation. Since the subgroup of $\operatorname{SL}(n, \mathbb{C})$ fixing the decomposition of $\left.V_{\rho}\right|_{Z}$ into isotypical components is a maximal torus, the image of $\rho$ is contained in the normalizer of a maximal torus. Furthermore, assume that $\rho$ underlies a VHS. The Galois action preserves the Hodge type, and there is a single Hodge type for the rank 1 isotypical component (note that the isotypical decomposition is compatible with the Hodge structure). Therefore $\rho$ has only a single Hodge type and it is unitary. This proves the lemma in the case of several isotypical components.

We may now assume $\left.V_{\rho}\right|_{Z}=V_{1} \otimes W_{1}$. Since the rank is $\leq 3$, and by hypothesis $\left.V_{\rho}\right|_{Z}$ is reducible so the rank of $V_{1}$ is strictly smaller than $n$, we get that $V_{1}$ has rank 1. It means that the restriction and projection to a representation $\pi_{1}(Z, z) \rightarrow$ $\operatorname{PGL}(n, \mathbb{C})$ is trivial. Since by hypothesis our representation is into $\operatorname{SL}(n, \mathbb{C})$ we get that $\rho$ has finite image. In particular it is unitary, and a unitary irreducible VHS can have only a single Hodge type. This completes the proof of the lemma.

COROLlARY 3.5. Let $X$ be a smooth complex projective variety, and suppose $\rho$ : $\pi_{1}(X, x) \rightarrow \mathrm{SL}(3, \mathbb{C})$ is an irreducible representation of rank 3 underlying a VHS with more than a single Hodge type. Then the conditions listed in Theorem 3.3 are equivalent.

Proof. By the above lemma, $\rho$ is not tensor decomposable and not virtually reducible.

\section{Structure of complex VHS of type $(1,1,1)$}

In this section we study general complex VHS of type $(1,1,1)$ on quasi-projective varieties. The main aim is to prove that every complex VHS of type $(1,1,1)$ or its dual can be constructed analogously to the following example:

Example 4.1. Let $X$ be a smooth complex projective surface. Let $L_{1}$ be a nef line bundle with strictly positive degree, $L_{1}^{2}=0$ and fixed inclusion $j_{1}: L_{1} \hookrightarrow \Omega_{X}$. Let us fix a line bundle $j_{2}: L_{2} \hookrightarrow \Omega_{X}$ generically the same as $j_{1}\left(L_{1}\right)$ such that $c_{1}\left(L_{2}\right)=a c_{1}\left(L_{1}\right)$ in $H^{2}(X, \mathbb{Q})$ for some (rational) $a \in\left(-\frac{1}{2}, 1\right]$. Finally, let us fix a line bundle $L_{0}$ such that $3 c_{1}\left(L_{0}\right)=(2+a) c_{1}\left(L_{1}\right)$ in $H^{2}(X, \mathbb{Q})$.

Let us consider the system of Hodge bundles defined by

$$
E^{2,0}:=L_{0}, \quad E^{1,1}:=L_{0} \otimes L_{1}^{-1}, \quad E^{0,2}:=L_{0} \otimes L_{1}^{-1} \otimes L_{2}^{-1}
$$


with $\theta$ given by inclusions $j_{i}: L_{i} \hookrightarrow \Omega_{X}$ tensored with identity on $E^{p, q}$ for $(p, q)=$ $(2,0),(1,1)$. Then for any ample line bundle $A$ the pair $(E, \theta)$ is an $A$-stable system of Hodge bundles with vanishing rational Chern classes.

Later we will see that if $a \neq 1$ then this system of Hodge bundles comes from an orbicurve (see Theorem 5.1).

Let $X$ be a smooth complex quasi-projective variety of dimension $d \geq 2$. Let us fix a smooth projective variety $\bar{X}$ containing $X$ as an open subset and such that $D=\bar{X}-X$ is a simple normal crossing divisor. In this section, we consider the quasi-projective case for future reference, although afterwards in the present paper we shall assume $D=\emptyset$.

Let $A$ be a fixed very ample divisor on $\bar{X}$. In the following we use the intersection pairing of $\mathbb{Q}$-divisors on the polarized variety $(\bar{X}, A)$ as defined in Subsection 2.2 .

Remark 4.2. Technically speaking, one should make a distinction between a line bundle and its corresponding divisor class. In the subsequent numerical calculations that would add burdensome extra notation, so we make the convention that a symbol such as, typically, $L_{i}$ can either mean the line bundle or the corresponding divisor class, according to context. In particular, we usually use the tensor product $L_{1} \otimes L_{2}$ but in computation of intersection numbers we use divisor classes and write, e.g., $\left(L_{1}+2 L_{2}\right)$. $A$ instead of $\left(L_{1} \otimes L_{2}^{\otimes 2}\right)$.A.

Let us recall that by Bertini's theorem the general complete intersection surface $\bar{Y}$ in $\bar{X}$ is smooth and irreducible. We can also assume that $D_{Y}=\bar{Y} \cap D$ is a simple normal crossing divisor on $\bar{Y}$. Let us set $Y:=\bar{Y}-D_{Y}$.

Let $\left(E=\bigoplus_{p+q=2} E^{p, q}, \theta\right)$ be a rank 3 logarithmic system of Hodge bundles of type $(1,1,1)$. Recall that this definition includes the condition that both KodairaSpencer maps $\theta$ be nonzero, so they induce injections of rank 1 sheaves

$$
L_{1}:=E^{2,0} \otimes\left(E^{1,1}\right)^{*} \hookrightarrow \Omega_{\bar{X}}(\log D)
$$

and

$$
L_{2}:=E^{1,1} \otimes\left(E^{0,2}\right)^{*} \hookrightarrow \Omega_{\bar{X}}(\log D) .
$$

The proof of the following proposition is inspired by the proof of [La1, Theorem 4.2].

Proposition 4.3. Assume that $E$ has vanishing rational Chern classes and that $(E, \theta)$ is slope A-stable. Then the following conditions are satisfied:

1. $c_{1}\left(E^{p, q}\right) \cdot c_{1}\left(E^{p^{\prime}, q^{\prime}}\right)=0$ for all pairs $(p, q)$ and $\left(p^{\prime}, q^{\prime}\right)$.

2. The classes of $L_{1}$ and $L_{2}$ lie on the same line in the vector space $H^{2}(\bar{X}, \mathbb{Q})$. 
3. There exist effective divisors $B_{1}$ and $B_{2}$ such that $M:=L_{1}\left(B_{1}\right)=L_{2}\left(B_{2}\right)$ is a saturation of both $L_{1}$ and $L_{2}$ in $\Omega_{\bar{X}}(\log D)$. Moreover, we have $L_{i} . M=0$ and $L_{i} \cdot B_{j}=0$ for all $i$ and $j$.

4. Either $\left.L_{1}\right|_{\bar{Y}}$ or $\left.L_{2}\right|_{\bar{Y}}$ is nef with self intersection zero and strictly positive degree.

Proof. We claim that the map

$$
\alpha: L_{1} \oplus L_{2} \rightarrow \Omega_{\bar{X}}(\log D)
$$

induced from $\theta$ has rank 1 . Indeed, in the local coordinates $\alpha$ is given by two logarithmic 1-forms $\omega_{1}$ and $\omega_{2}$ and the integrability of $\theta$ implies that $\omega_{1} \wedge \omega_{2}=0$, so they are proportional and $\alpha$ has rank 1. Let $M$ be the saturation of the image of $\alpha$ in $\Omega_{\bar{X}}(\log D)$ (i.e., the largest rank 1 subsheaf of $\Omega_{\bar{X}}(\log D)$ containing the image of $\alpha$ ). Then $M$ is a line bundle (as it is a rank 1 reflexive $\mathscr{O}_{X}$-module on a smooth variety) such that $\Omega_{\bar{X}}(\log D) / M$ is torsion free.

By construction we have inclusions $L_{1} \subset M$ and $L_{2} \subset M$ defining effective divisors $B_{1}$ and $B_{2}$, respectively. Let us set $e^{p, q}=c_{1}\left(E^{p, q}\right), l_{i}=c_{1}\left(L_{i}\right)$ and $b_{i}=$ $c_{1}\left(B_{i}\right)$. Then we can write

$$
m=c_{1}(M)=l_{1}+b_{1}=l_{2}+b_{2} .
$$

By assumption we have

$$
e^{0,2}+e^{1,1}+e^{2,0}=0
$$

in the rational cohomology $H^{2}(\bar{X}, \mathbb{Q})$, so the classes $e^{p, q}$ can be written in terms of $l_{1}$ and $l_{2}$ in the following way:

$$
e^{2,0}=\frac{1}{3}\left(2 l_{1}+l_{2}\right), \quad e^{1,1}=\frac{1}{3}\left(l_{2}-l_{1}\right), \quad e^{0,2}=\frac{1}{3}\left(-l_{1}-2 l_{2}\right) .
$$

Therefore

$$
3 m=l_{1}+b_{1}+2\left(l_{2}+b_{2}\right) \geq l_{1}+2 l_{2}=-3 e^{0,2} .
$$

In particular, we have $m \geq-e^{0,2}$. Similarly, we have

$$
3 m=2\left(l_{1}+b_{1}\right)+\left(l_{2}+b_{2}\right) \geq 2 l_{1}+l_{2}=3 e^{2,0},
$$

so $m \geq e^{2,0}$.

The following lemma is a corollary of the well-known Bogomolov's lemma:

LEMMA 4.4. For any line bundle $N \subset \Omega_{\bar{X}}(\log D)$ the restriction $\left.N\right|_{\bar{Y}}$ is not big on $\bar{Y}$. 
Proof. We have a short exact sequence

$$
\left.0 \rightarrow \mathscr{O}_{\bar{Y}}(-A)^{n-2} \rightarrow \Omega_{\bar{X}}(\log D)\right|_{\bar{Y}} \rightarrow \Omega_{\bar{Y}}\left(\log D_{Y}\right) \rightarrow 0 .
$$

Let us consider the composition $\left.\left.N\right|_{\bar{Y}} \rightarrow \Omega_{\bar{X}}(\log D)\right|_{\bar{Y}} \rightarrow \Omega_{\bar{Y}}\left(\log D_{Y}\right)$. By Bogomolov's lemma (see [Bo, Theorem 4] and [EV] Corollary 6.9]) $\Omega_{\bar{Y}}\left(\log D_{Y}\right)$ does not contain big line bundles. So if this composition is non-zero then $\left.N\right|_{\bar{Y}}$ is not big.

If the map $\left.N\right|_{\bar{Y}} \rightarrow \Omega_{\bar{Y}}\left(\log D_{Y}\right)$ is zero then by the above exact sequence $\left.N\right|_{\bar{Y}}$ is contained in $\mathscr{O}_{\bar{Y}}(-A)^{n-2}$, so $\kappa\left(\left.N\right|_{\bar{Y}}\right)=-\infty$.

Slope $A$-stability of $(E, \theta)$ implies that

$$
e^{0,2} \cdot A<0
$$

and

$$
-e^{2,0} \cdot A=\left(e^{0,2}+e^{1,1}\right) \cdot A<0 .
$$

If $\left(e^{0,2}\right)^{2}>0$ then, thanks to Lemma 2.3 , the first inequality implies that $-\left.e^{0,2}\right|_{\bar{Y}}$ is big. But then $\left.M\right|_{\bar{Y}}$ is big, which contradicts the above lemma. Therefore

$$
\left(e^{0,2}\right)^{2} \leq 0
$$

Similarly, if $\left(e^{2,0}\right)^{2}>0$ then $\left.e^{2,0}\right|_{\bar{Y}}$ is big. But then $\left.M\right|_{\bar{Y}}$ is big, a contradiction. Therefore

$$
\left(e^{2,0}\right)^{2} \leq 0
$$

Let us also recall that

$$
0=c_{2}(E)=e^{2,0} \cdot e^{1,1}+e^{1,1} \cdot e^{0,2}+e^{0,2} \cdot e^{2,0}=-\left(e^{2,0}\right)^{2}+e^{1,1} \cdot e^{0,2}
$$

Therefore

$$
L_{2}^{2}=\left(e^{1,1}+e^{0,2}\right)^{2}-4 e^{1,1} \cdot e^{0,2}=\left(e^{2,0}\right)^{2}-4\left(e^{2,0}\right)^{2}=-3\left(e^{2,0}\right)^{2} \geq 0 .
$$

Similarly, we have

$$
L_{1}^{2}=\left(e^{2,0}-e^{1,1}\right)^{2}=-3\left(e^{0,2}\right)^{2} \geq 0 .
$$

From stability of $E$ we get $\left(L_{1}+2 L_{2}\right) \cdot A=-3 e^{0,2} \cdot A>0$ and hence there exists $i$ with $L_{i} . A>0$. Lemma 2.3 and Lemma 4.4 imply that $\left.L_{i}\right|_{\bar{Y}}$ is nef with $L_{i}^{2}=0$ and the degree of $\left.L_{i}\right|_{\bar{Y}}$ is strictly positive.

LEMMA 4.5. We have $L_{1}^{2}=L_{1} \cdot L_{2}=L_{2}^{2}=0$. 
Proof. Let us first assume that $i=1$, i.e., $\left.L_{1}\right|_{\bar{Y}}$ is nef with $L_{1}^{2}=0$. Since $B_{2}$ is effective, we have $L_{1} \cdot B_{2} \geq 0$. By Lefschetz' hyperplane theorem for quasi-projective varieties (see [HL, Theorem 1.1.3]) we have $\pi_{1}(Y) \simeq \pi_{1}(X)$. So by functoriality of the correspondence between representations and Higgs bundles, the restriction

$$
\left(\left.E\right|_{\bar{Y}}, \theta_{Y}:\left.\left.\left.\left.E\right|_{\bar{Y}} \stackrel{\left.\theta\right|_{Y}}{\rightarrow} E\right|_{\bar{Y}} \otimes \Omega_{\bar{X}}(\log D)\right|_{\bar{Y}} \rightarrow E\right|_{\bar{Y}} \otimes \Omega_{\bar{Y}}\left(\log D_{Y}\right)\right)
$$

of $(E, \theta)$ to $\bar{Y}$ is $A$-stable (one can also give a direct proof of this fact: see [La2, Theorem 12] and [La3] $)$. Since the rational Chern classes of $\left.E\right|_{\bar{Y}}$ vanish, $\left(\left.E\right|_{\bar{Y}}, \theta_{Y}\right)$ is stable with respect to every stable polarization and semistable with respect to every nef polarization. In particular, $\left.L_{1}\right|_{\bar{Y}}$-semistability of $\left(\left.E\right|_{\bar{Y}}, \theta_{Y}\right)$ implies that $L_{1} .\left(L_{1}+2 L_{2}\right) \geq 0$. Therefore we have $L_{1} . L_{2} \geq 0$.

Applying Lemma2.4 to $M=L_{1}+B_{1}$ we see that $L_{1}^{2}=0, L_{1} \cdot B_{1}=0$ and $L_{1} \cdot M=$ 0 . Using $M=L_{2}+B_{2}$ we get equalities $L_{1} \cdot L_{2}=L_{1} \cdot B_{2}=0$. But then

$$
c_{2}(E)=\frac{1}{9}\left(L_{1}^{2}+L_{1} \cdot L_{2}+L_{2}^{2}\right)=0
$$

implies that $L_{2}^{2}=-L_{1} \cdot L_{2}=0$.

The proof in the case $i=2$ is analogous.

This lemma finishes the proof of assertions (1) and (4) of the proposition. To prove (2), let us consider $i^{\prime}$ such that $\left\{i, i^{\prime}\right\}=\{1,2\}$. Let us choose a rational $a$ such that $\left(L_{i^{\prime}}-a L_{i}\right) \cdot A=0$. Since $\left(L_{i^{\prime}}-a L_{i}\right)^{2}=0$ this implies that $l_{i^{\prime}}=a l_{i}$ in $H^{2}(X, \mathbb{Q})$.

Assertion (3) of the proposition follows from the proof of Lemma 4.5. More precisely, if $i=1$ then this proof shows that $L_{1} \cdot M=L_{1} \cdot B_{1}=L_{1} \cdot B_{2}=0$. So (2) implies that $L_{2} \cdot M=L_{2} \cdot B_{1}=L_{2} \cdot B_{2}=0$. The proof in case $i=2$ is analogous.

Remark 4.6. Let us remark that stability of $E$ implies that $\left(L_{1}+2 L_{2}\right) \cdot A>0$ and $\left(2 L_{1}+L_{2}\right) . A>0$ so that $a>-\frac{1}{2}$ in the notation of the above proof. In fact, this condition is sufficient to define a stable rank 3 logarithmic system of Hodge bundles.

Let us recall that a smooth log pair is a pair consisting of a smooth variety and a (reduced) simple normal crossing divisor. A morphism of log pairs $\bar{f}:\left(\bar{Z}, D_{Z}\right) \rightarrow(\bar{X}, D)$ is a proper morphism of normal varieties such that $\bar{f}\left(D_{Z}\right)=D$. For a morphism of smooth projective $\log$ pairs $\bar{f}:\left(\bar{Z}, D_{Z}\right) \rightarrow(\bar{X}, D)$, we write $Z:=\bar{Z}-D_{Z}$, we choose a point $z$ over $x$ and we set $f=\left.\bar{f}\right|_{\bar{Z}-D_{Z}}$.

Let $\rho: \pi_{1}(X, x) \rightarrow \operatorname{SL}(n, \mathbb{C})$ be an irreducible representation with quasi-unipotent monodromy at infinity (i.e., such that $\rho$ has quasi-unipotent monodromies along small simple loops around all irreducible components of $D$ ). Then by Kawamata's covering trick there exists a finite flat morphism of smooth projective log 
pairs $\bar{f}:\left(\bar{Z}, D_{Z}\right) \rightarrow(\bar{X}, D)$ such that $\left(\bar{f}^{*} D\right)_{\text {red }}=D_{Z}$ and $f^{*} \rho: \pi_{1}(Z, z) \rightarrow \operatorname{SL}(n, \mathbb{C})$ has unipotent monodromy at infinity, i.e., $f^{*} \rho$ has unipotent local monodromies along all irreducible components of $D_{Z}$. This implies that the residues of Deligne's canonical extension of the flat bundle associated to $f^{*} \rho$ are nilpotent (since all the eigenvalues of the residues are zero) and we are interested in bundles with trivial parabolic structure.

More precisely, let us consider a vector bundle with connection $(V, \nabla)$ with regular singularities and rational residues in $[0,1)$ along the irreducible components of $D$, that corresponds to the representation $\rho$. This bundle comes equipped with a canonical parabolic structure, which makes the corresponding parabolic flat bundle stable with vanishing parabolic Chern classes (cf. [Mo1], [Mo2] and [IS, Lemma 3.3]).

If we assume the local system $V_{\rho}$ underlies a complex variation of Hodge structure then $f^{*} \rho$ also underlies a complex VHS. The logarithmic parabolic Higgs bundle corresponding to $\rho$ is stable and it has vanishing parabolic first and second Chern classes (see [Mo1] and [Mo2] $)$. The pullback of this logarithmic parabolic Higgs bundle to $\left(\bar{Z}, D_{Z}\right)$ (cf. [IS, Lemma 3.7]) becomes a stable logarithmic system of Hodge bundles (with trivial parabolic structure) which has vanishing all rational Chern classes (by functoriality of the Kobayashi-Hitchin correspondence, the Higgs bundle associated to the pull back $f^{*} \rho$ corresponds to a complex VHS, so it is a system of Hodge bundles). Thus we have the following lemma:

LEMMA 4.7. Let $\rho: \pi_{1}(X, x) \rightarrow \mathrm{SL}(n, \mathbb{C})$ be an irreducible representation with quasi-unipotent monodromy at infinity. If $\rho$ underlies a complex VHS then there exists a finite flat morphism of smooth projective log pairs $\bar{f}:\left(\bar{Z}, D_{Z}\right) \rightarrow(\bar{X}, D)$ such that $f^{*} \rho: \pi_{1}(Z, z) \rightarrow \mathrm{SL}(n, \mathbb{C})$ has unipotent monodromy at infinity. To this representation the Kobayashi-Hitchin correspondence associates a stable rank $n$ system of logarithmic Hodge sheaves with vanishing rational Chern clases.

In case the underlying rank 3 logarithmic system of Hodge bundles on $\left(\bar{Z}, D_{Z}\right)$ is of type $(1,1,1)$, we can apply Proposition 4.3 .

Remark 4.8. If $\rho$ is a rigid irreducible representation with quasi-unipotent monodromy at infinity, then it underlies a complex VHS (this part of [CS, Theorem 8.1] works in any rank) and we can apply the above lemma to such representations.

\section{Geometry of complex VHS of type $(1,1,1)$}

Let $X$ be a smooth complex projective variety. If $\rho: \pi_{1}(X, x) \rightarrow \operatorname{SL}(n, \mathbb{C})$ is a representation then the induced projective representation $\pi_{1}(X) \rightarrow \operatorname{PGL}(n, \mathbb{C})$ is denoted by $\bar{\rho}$. 
THEOREM 5.1. Let $\rho: \pi_{1}(X, x) \rightarrow \mathrm{SL}(3, \mathbb{C})$ be an irreducible representation coming from a complex variation of Hodge structure of type $(1,1,1)$. Let $V_{\rho}$ denote the corresponding local system. Then one of the following holds:

1. There exists a projective representation $\pi_{1}(X, x) \rightarrow \operatorname{PGL}(2, \mathbb{C})$ which induces $\bar{\rho}$ via the homomorphism $\operatorname{PGL}(2, \mathbb{C}) \rightarrow \operatorname{PGL}(3, \mathbb{C})$ given by second symmetric power. Moreover, there exists a finite covering $\pi: Y \rightarrow X$ from a smooth projective variety $Y$, a rank 1 local system $W_{1}$ on $X$ such that $W_{1}^{\otimes 3}$ is trivial and a rank 2 local system $W_{2}$ on $Y$ such that $\pi^{*} V_{\rho}=\pi^{*} W_{1} \otimes \operatorname{Sym}^{2} W_{2}$.

2. $\rho$ projectively factors through an orbicurve.

Proof. We can assume that $X$ has dimension at least 2 as otherwise there is nothing to be proven. Therefore the complex variation of Hodge structure corresponding to $\rho$ is of the form described in Proposition 4.3. Let us write $L_{2} \equiv a L_{1}$. Since the assertion for a representation is equivalent to the assertion for its dual, we can assume that for a general complete intersection surface $S \subset X$ the restriction $\left.L_{1}\right|_{S}$ is nef with strictly positive degree and $a \leq 1$.

In the notation of Proposition 4.3 we have $M=L_{1}\left(B_{1}\right)=L_{2}\left(B_{2}\right)$. Let us write $B_{1}=B+B_{1}^{\prime}$ and $B_{2}=B+B_{2}^{\prime}$, where $B, B_{1}^{\prime}, B_{2}^{\prime}$ are effective and $B_{1}^{\prime}$ and $B_{2}^{\prime}$ have no common irreducible components. Recall that $L_{1} \cdot B=L_{1} \cdot B_{1}^{\prime}=0$ and $L_{2} \cdot B=$ $L_{2} . B_{2}^{\prime}=0$. We set $M^{\prime}:=M(-B)$. This line bundle has a canonical inclusion into $\Omega_{X}$ given by composing $M(-B) \subset M$ with $M \rightarrow \Omega_{X}$. By definition we also have $M^{\prime}=L_{1}\left(B_{1}^{\prime}\right)=L_{2}\left(B_{2}^{\prime}\right)$. Intersecting both sides of equality $L_{1}+B_{1}^{\prime}=L_{2}+B_{2}^{\prime}$ with $B_{1}^{\prime}$ we get

$$
\left(B_{1}^{\prime}\right)^{2}=B_{1}^{\prime} \cdot B_{2}^{\prime} \geq 0
$$

(here we use that $L_{2} \cdot B_{1}^{\prime}=a L_{1} \cdot B_{1}^{\prime}=0$, and since $B_{1}^{\prime}$ and $B_{2}^{\prime}$ have no common components their intersection is positive). But Lemma 2.4 implies that $\left(B_{1}^{\prime}\right)^{2} \leq 0$, so we have $\left(B_{1}^{\prime}\right)^{2}=B_{1}^{\prime} \cdot B_{2}^{\prime}=0$. Hence we also get $\left(B_{2}^{\prime}\right)^{2}=0$. There exists also some non-negative $\alpha_{1}$ such that $\left(B_{1}^{\prime}-\alpha_{1} L_{1}\right) \cdot A=0$. Since $\left(B_{1}^{\prime}-\alpha_{1} L_{1}\right)^{2}=0$, we have $B_{1}^{\prime} \equiv \alpha_{1} L_{1}$. Similarly, there exists some non-negative $\alpha_{2}$ such that $B_{2}^{\prime} \equiv \alpha_{2} L_{1}$. Note also that $B_{i}^{\prime}=0$ is equivalent to $\alpha_{i}=0$, since if $B_{i}^{\prime}$ is a non-zero effective divisor then $B_{i}^{\prime} \cdot A>0$. Let us recall that $L_{1}+B_{1}^{\prime}=L_{2}+B_{2}^{\prime}$, so $\left(1+\alpha_{1}\right) L_{1} \equiv\left(a+\alpha_{2}\right) L_{1}$. But $L_{1} . A>0$, so $1+\alpha_{1}=a+\alpha_{2}$. Since $a \leq 1$, this implies that $0 \leq \alpha_{1} \leq \alpha_{2}$.

We consider two cases depending on whether $B_{2}^{\prime}$ is zero or not.

If $B_{2}^{\prime}=0$ then $\alpha_{2}=\alpha_{1}=0, B_{1}^{\prime}=0$ and $L:=L_{1}=L_{2}$ with the same map to $M$ and $\Omega_{X}$. In this case we set $N:=E^{2,0} \otimes L_{1}^{-1}$. Since $\operatorname{det} E=N^{\otimes 3}$, the line bundle $N$ is 3-torsion. Therefore $(E, \theta) \simeq(N, 0) \otimes \operatorname{Sym}^{2}(F) \otimes \operatorname{det}(F)^{-1}$, where $F=\left(F^{1,0} \oplus\right.$ $\left.F^{0,1}, \theta_{F}\right)$ is a system of Hodge bundles with $F^{1,0}=L, F^{0,1}=\mathscr{O}_{X}$ and the Higgs field $\theta_{F}$ given by the inclusion $L \rightarrow \Omega_{X}$. Note that $F$ is an $A$-stable system of 
Hodge bundles but it does not have vanishing Chern classes so it does not underlie a rank 2 representation of $\pi_{1}(X, x)$. But it comes from a projective representation $\pi_{1}(X, x) \rightarrow$ PGL $(2, \mathbb{C})$. There exists a finite covering $\pi: Y \rightarrow X$ such that $\pi^{*} L=$ $M^{\otimes 2}$ for some line bundle $M$ on $Y$. Then $\pi^{*}(E, \theta) \simeq \pi^{*}(N, 0) \otimes \operatorname{Sym}^{2}\left(F_{Y}\right)$, where $F_{Y}=\left(F_{Y}^{1,0} \oplus F_{Y}^{1,0}, \theta_{Y}\right)$ is a system of Hodge bundles with $F_{Y}^{1,0}=M, F_{Y}^{0,1}=M^{-1}$ and the Higgs field $\theta_{Y}$ given by the inclusion $\pi^{*} L \rightarrow \pi^{*} \Omega_{X} \rightarrow \Omega_{Y}$.

This corresponds to case (1) of the theorem.

If $B_{2}^{\prime} \neq 0$, then by the Hodge index theorem there exist positive integers $b$ and $c$ such that $b M^{\prime} \equiv c B_{2}^{\prime}$. The idea is now to say that $M^{\prime}$ looks like an effective divisor. Roughly speaking, we try to get a section of $M^{\prime}$ and then argue using this differential form. In practice we need to extract a root, and we only have numerical information so, in the case where the Picard scheme has positive dimension, we need to deal with several cases.

Start by noting that $U:=b M^{\prime}-c B_{2}^{\prime} \in \operatorname{Pic}^{\tau}(X)$. Multiplying $b$ and $c$ by the same positive integer we can assume that in fact $U \in \operatorname{Pic}^{0}(X)$. Therefore we have a nonzero map

$$
U \rightarrow\left(M^{\prime}\right)^{\otimes b}
$$

determined by the divisor $c B_{2}^{\prime}$. We can write $U=V^{\otimes b}$ for some $V \in \operatorname{Pic}^{0}(X)$ and hence we get a section $\eta \in H^{0}\left(X,\left(M^{\prime} \otimes V^{*}\right)^{\otimes b}\right)$ (whose divisor is $c B_{2}^{\prime}$ ).

Let $p: Z \rightarrow X$ be a desingularization of the ramified covering defined by taking the $b$-th root of $\eta$. Over $Z$ we have a tautological section $\alpha \in H^{0}\left(Z, p^{*}\left(M^{\prime} \otimes V^{*}\right)\right)$ such that $\alpha^{\otimes b}=p^{*} \eta$.

In particular, $\alpha \in H^{0}\left(Z, \Omega_{Z}^{1} \otimes p^{*} V^{*}\right)$, which means that $p^{*} V^{*} \in \operatorname{Pic}^{0}(Z)$ is in the jump-locus for twisted sections of $\Omega_{Z}^{1}$ :

$$
S(Z):=\left\{N \in \operatorname{Pic}^{0}(Z) \mid \operatorname{dim} H^{0}\left(Z, \Omega_{Z}^{1} \otimes N\right) \geq 1\right\} .
$$

The irreducible components of this jump-locus are translates of abelian subvarieties of $\mathrm{Pic}^{0}(Z)$ by torsion points. The fact that the components are translates of abelian varieties was proved in [GL] and the assertion about torsion was proved in [Si3]. We distinguish two cases depending on whether $p^{*} V^{*}$ is a torsion point or not.

Case 1. Let us assume that $p^{*} V^{*}$ is a torsion point of $\operatorname{Pic}^{0}(Z)$.

Then the line bundle $p^{*} V^{*}$ defines a finite étale covering $q: Z^{\prime} \rightarrow Z$ such that $V^{*}$ becomes trivial after pulling back to $Z^{\prime}$. Let $p^{\prime}$ denote the composition of $q: Z^{\prime} \rightarrow Z$ with $p: Z \rightarrow X$. Then over $Z^{\prime}$ we get a nonzero section $\xi \in H^{0}\left(Z^{\prime}, \Omega_{Z^{\prime}}^{1}\right)$ given by the composition of $q^{*} \alpha: \mathscr{O}_{Z^{\prime}} \rightarrow q^{*}\left(p^{*}\left(M^{\prime} \otimes V^{*}\right)\right) \simeq q^{*}\left(p^{*}\left(M^{\prime}\right)\right)=\left(p^{\prime}\right)^{*} M^{\prime}$ with the canonical map $\left(p^{\prime}\right)^{*} M^{\prime} \rightarrow\left(p^{\prime}\right)^{*} \Omega_{X} \rightarrow \Omega_{Z^{\prime}}^{1}$.

The line bundle $\left(p^{\prime}\right)^{*} M^{\prime} \subset \Omega_{Z^{\prime}}^{1}$ is generically generated by this section $\xi$. 
We can look at the Albanese map defined by $\xi$ (see [Si2, p. 101]). This is the map $\psi: Z^{\prime} \rightarrow A=\operatorname{Alb}\left(Z^{\prime}\right) / B$, where $B$ is the sum of all abelian subvarieties of $\operatorname{Alb}\left(Z^{\prime}\right)$ on which $\xi$ vanishes. By construction $\xi=\psi^{*}\left(\xi_{A}\right)$ for some 1 -form $\xi_{A}$ on $A$ such that the restriction of $\xi_{A}$ to any nontrivial abelian subvariety of $A$ is nonzero.

We distinguish two subcases depending on the dimension of $\psi\left(Z^{\prime}\right)$ :

Subcase 1.1. The image of $\psi$ is a curve.

In this case taking the Stein factorization of $Z^{\prime} \rightarrow \psi\left(Z^{\prime}\right)$, we get a fibration $f: Z^{\prime} \rightarrow C$ over a smooth projective curve and a 1 -form $\xi_{C}$ on $C$ such that $\xi=f^{*} \xi_{C}$. Considering $\xi$ as a map $\mathscr{O}_{Z^{\prime}} \rightarrow \Omega_{Z^{\prime}}$, this means that the map factors through $f^{*} \xi_{C}$ : $\mathscr{O}_{Z^{\prime}}=f^{*} \mathscr{O}_{C} \rightarrow f^{*} \Omega_{C}$. Therefore $\left(p^{\prime}\right)^{*} M$ is contained in the saturation of $f^{*} \Omega_{C}$ in $\Omega_{Z^{\prime}}$. If $F$ is a smooth fiber of $f$ (or just a multiplicity 1 irreducible component of a fiber of $f$ ) then this shows that the canonical map $\left.\left.\left(p^{\prime}\right)^{*} M\right|_{F} \rightarrow \Omega_{Z^{\prime} / C}\right|_{F}=\Omega_{F}$ is zero. This implies that the Kodaira-Spencer maps of our variation of Hodge structures restricted to the fiber $F$, vanish. Each Hodge subbundle is therefore a flat subbundle. Hence the restriction of our variation of Hodge structures to $F$ splits into a direct sum of three rank 1 variations of Hodge structure at the three different Hodge types. Clearly, $\rho$ is not virtually tensor decomposable. It is also not virtually reducible as the Kodaira-Spencer maps remain nonzero under alterations. So by Theorem $3.3(4 \Rightarrow 1), \rho$ projectively factors through an orbicurve.

Subcase 1.2. The dimension of the image $\psi\left(Z^{\prime}\right)$ is at least 2 .

Let $\tilde{A} \rightarrow A$ be the universal covering of $A$ (treated as a complex vector space). This map is only analytic and not algebraic. Let $\tilde{Z}:=Z^{\prime} \times_{A} \tilde{A}$ be the covering of $Z^{\prime}$ defined by the Albanese map $\psi$ and let $\pi: \tilde{Z} \rightarrow Z^{\prime}$ be the projection on the first factor and $\tilde{\psi}: \tilde{Z} \rightarrow \tilde{A}$ on the second factor (these maps are also only analytic). Let us fix a point $\tilde{z}_{0} \in \tilde{Z}$. There exists a unique linear function $g_{\tilde{A}}: \tilde{A} \rightarrow \mathbb{C}$ such that $g_{\tilde{A}}\left(\tilde{\psi}\left(\tilde{z}_{0}\right)\right)=0$ and $d g_{\tilde{A}}=\xi_{A}$. Let $g: \tilde{Z} \rightarrow \mathbb{C}$ be the composition of $g_{\tilde{A}}$ with $\tilde{\psi}$. Then the Lefschetz theorem (see [Si2, Theorem 1]; since $\operatorname{dim}\left(\psi\left(Z^{\prime}\right)\right) \geq 2$ the assumptions of this theorem are satisfied) says that for any fibre $F$ of $g$ (or in other words a possibly non-compact leaf of the foliation defined by $\xi$ ), the map $\pi_{1}\left(F, \tilde{z}_{0}\right) \rightarrow \pi_{1}\left(\tilde{Z}, \tilde{z}_{0}\right)$ is surjective.

Note that by our construction the restriction $\left.\pi^{*} \xi\right|_{F}$ is zero. Choosing $F$ so that it does not lie in the preimage of $B_{2}^{\prime}$, the inclusion $M^{\prime} \rightarrow \Omega_{X}$ becomes zero after pulling back to $F$ and composing with the canonical map to $\Omega_{F}$. So our variation of Hodge structure splits on $F$ into a direct sum of three line bundles. In this case the image of the representation $\pi_{1}\left(F, \tilde{z}_{0}\right) \rightarrow \operatorname{SL}(3, \mathbb{C})$ is contained in a maximal torus. Therefore the monodromy representation is abelian on $\pi_{1}\left(\tilde{Z}, \tilde{z}_{0}\right)$. But $\pi_{1}\left(Z^{\prime}, \tilde{\psi}\left(\tilde{z}_{0}\right)\right) / \pi_{1}\left(\tilde{Z}, \tilde{z}_{0}\right)$ is an abelian group, so the monodromy of our original VHS would be solvable. Then the Zariski closure $G \subset \operatorname{SL}(3, \mathbb{C})$ of im $\rho$ is also 
solvable (this follows from [Bor, I.2.4 Proposition]). On the other hand, since $\rho$ is irreducible, the connected component $G^{0}$ is reductive. Hence $G^{0}$ is contained in a maximal torus of SL $(3, \mathbb{C})$. Taking the étale covering $q: \tilde{X} \rightarrow X$ corresponding to the image of $\pi_{1}(X, x) \rightarrow G / G^{0}$, we see that the monodromy of $q^{*} V_{\rho}$ is contained in the maximal torus of $\operatorname{SL}(3, \mathbb{C})$. But then the corresponding pull-back of the system of Hodge bundles is a direct sum of line bundles with trivial maps between the components, contradicting the fact that the original VHS was of type $(1,1,1)$.

Case 2. Assume that $p^{*} V^{*}$ is not a torsion point.

Then an irreducible component of $S(Z)$ containing this point has dimension $\geq 1$.

Let us recall that for a line bundle $N \in \operatorname{Pic}^{0}(Z)$ we have an isomorphism of complex vector spaces

$$
H^{0}\left(Z, \Omega_{Z}^{1} \otimes N\right) \simeq \overline{H^{1}\left(Z, N^{-1}\right)}
$$

so

$$
S(Z)=\left\{N \in \operatorname{Pic}^{0}(Z) \mid \operatorname{dim} H^{1}\left(Z, N^{-1}\right) \geq 1\right\} .
$$

Beauville proves in $[\mathrm{Be}]$ that the positive-dimensional components of this set all come from maps to orbicurves. More precisely, [Be, Corollaire 2.3] says that there exists a fibration $f: Z \rightarrow C$ over a smooth projective curve of genus $g \geq 1$ such that $p^{*} V^{*}$ is trivial on every smooth fiber of $f$.

The proof of the following lemma is modelled on the proof of [Be, Proposition $1.10]$.

LEMMA 5.2. Let $Z$ be a smooth complex projective variety and let $C$ be a smooth complex projective curve. Let $f: Z \rightarrow C$ be a fibration with reduced fibers. Let $L \in \operatorname{Pic}^{\tau} Z$ be a non-torsion line bundle, which is trivial on some smooth fiber of f. If $0 \neq \alpha \in H^{0}\left(\Omega_{Z} \otimes L^{-1}\right)$ then there exists a line bundle $L_{C}$ on $C$ and $\beta \in$ $H^{0}\left(\Omega_{C} \otimes L_{C}^{-1}\right)=\operatorname{Hom}\left(L_{C}, \Omega_{C}\right)$ such that $L=f^{*} L_{C}$ and the composition

$$
L=f^{*} L_{C} \stackrel{f^{*} \beta}{\longrightarrow} f^{*} \Omega_{C} \longrightarrow \Omega_{Z}
$$

corresponds to $\alpha$.

Proof. First let us recall that existence of $L_{C}$ such that $L=f^{*} L_{C}$ follows from $[\mathrm{Be}$, Proposition 1.2]. We have a short exact sequence

$$
0 \rightarrow H^{1}\left(C, L_{C}\right) \rightarrow H^{1}(Z, L) \rightarrow H^{0}\left(C, R^{1} f_{*} L\right) \rightarrow 0
$$

coming from the Leray spectral sequence. Using the isomorphisms $H^{1}(Z, L) \simeq$ $\overline{H^{0}\left(Z, \Omega_{Z}^{1} \otimes L^{-1}\right)}$ and $H^{1}\left(C, L_{C}\right) \simeq \overline{H^{0}\left(C, \Omega_{C}^{1} \otimes L_{C}^{-1}\right)}$, it is sufficient to show that 
$H^{0}\left(C, R^{1} f_{*} L\right)=0$. But $H^{0}\left(C, R^{1} f_{*} L\right)=H^{0}\left(C, L_{C} \otimes R^{1} f_{*} \mathscr{O}_{Z}\right)$ and $R^{1} f_{*} \mathscr{O}_{Z}$ is dual to $f_{*} \omega_{Z / C}$, so we need to check that $\operatorname{Hom}\left(f_{*} \omega_{Z / C}, L_{C}\right)=0$. Let us recall that by [Fu, Theorem] (see also [CD, Theorem 17]), $f_{*} \omega_{Z / C}$ is a direct sum of an ample vector bundle and a direct sum of stable vector bundles of degree zero. Therefore $\operatorname{Hom}\left(f_{*} \omega_{Z / C}, L_{C}\right) \neq 0$ if and only if $L_{C}$ is isomorphic to one of the direct summands of $f_{*} \omega_{Z / C}$. But by Deligne's result, the rank 1 summands of $f_{*} \omega_{Z / C}$ are torsion (see [CD, Corollary 21]) and $L_{C}$ is not torsion, so we get a contradiction.

Taking a semistable reduction of $f$ we can assume that all the fibres of $f$ are reduced. Then, by the above lemma, there exists a line bundle $L_{C} \in \operatorname{Pic}^{0}(C)$ such that $p^{*} V=f^{*} L_{C}$ and the section $\alpha$ comes from a section of $H^{0}\left(C, \Omega_{C}^{1} \otimes L_{C}^{*}\right)$. This shows that $p^{*} V^{*} \rightarrow f^{*} \Omega_{C}$ is an isomorphism at the generic point of $Z$. Since $p^{*} V \rightarrow$ $p^{*} M$ is also an isomorphism at the generic point of $Z$, the sheaf $p^{*} M$ is contained in $f^{*} \Omega_{C} \subset \Omega_{Z}$ (since the fibres of $f$ are reduced, $f^{*} \Omega_{C}$ is saturated in $\Omega_{Z}$ ).

As before this implies that the pull back of our variation of Hodge structure to a fibre of $f$ splits into a direct sum of three rank 1 variations of Hodge structure. Therefore Corollary 3.5 implies that $\rho$ projectively factors through an orbicurve. Alternatively, the same arguments as before show that $\left(\left(p^{\prime}\right)^{*} M^{\prime}\right)^{\otimes b}$ is a line bundle associated to a certain sum of rational multiples of fibers of $f$ (but not necessarily positive). Then by passing to a certain cover $Z^{\prime \prime} \rightarrow Z^{\prime}$ we conclude that the pull back of our original variation of Hodge structure to $Z^{\prime \prime}$ is isomorphic to the pull back of a variation of Hodge structure on a certain curve $C^{\prime}$. So $\rho$ virtually projectively factors through an orbicurve and we can again conclude by Corollary 3.5. This completes the proof of Theorem 4.1.

\section{Some remarks on the case $(1,1, \ldots, 1)$}

In this section we consider the possibility of generalisation of the results of Section 4 to the case $(1,1, \ldots, 1)$. For simplicity of notation, we consider only the case when $X$ is a smooth projective variety (i.e., $\bar{X}=X$ ).

Let $n$ be a positive integer and let $\left(E=\bigoplus_{p+q=n} E^{p, q}, \theta\right)$ be a rank $(n+1)$ system of Hodge bundles of type $(1, \ldots, 1)$. Then $\theta$ induces injections

$$
L_{i}:=E^{n-i+1, i-1} \otimes\left(E^{n-i, i}\right)^{*} \hookrightarrow \Omega_{X}
$$

for $i=1, \ldots, n$.

Lemma 6.1. Assume that $c_{1}(E)=0$ and $(E, \theta)$ is slope A-stable. Let us set

$$
N_{i}:=(n-i+1)\left(L_{1}+2 L_{2}+\ldots+i L_{i}\right)+i\left((n-i) L_{i+1}+\ldots+2 L_{n-1}+L_{n}\right)
$$


for $i=1, \ldots, n$. Then for all non-negative real numbers $x_{1}, \ldots, x_{n}$ we have

$$
\left(\sum_{i=1}^{n} x_{i} N_{i}\right)^{2} \leq 0 .
$$

Proof. We claim that for all $i=1, \ldots, n-1$ the map

$$
\alpha_{i}: L_{i} \oplus L_{i+1} \rightarrow \Omega_{X}
$$

induced from $\theta$ has rank 1. Indeed, in the local coordinates $\alpha$ is given by two 1 -forms $\omega_{1}$ and $\omega_{2}$ and the integrability of $\theta$ implies that $\omega_{1} \wedge \omega_{2}=0$, so they are proportional and $\alpha_{i}$ has rank 1. It follows that the map

$$
\alpha: \bigoplus_{i=1}^{n} L_{i} \rightarrow \Omega_{X}
$$

also has rank 1. Let $M$ be the saturation of the image of $\alpha$ in $\Omega_{X}$ (i.e., the largest rank 1 subsheaf of $\Omega_{X}$ containing the image of $\alpha$ ). Then $M$ is a line bundle such that $\Omega_{X} / M$ is torsion free.

By construction we have inclusions $L_{i} \subset M$ defining effective divisors $B_{i}$. Let us set $e^{p, q}=c_{1}\left(E^{p, q}\right), l_{i}=c_{1}\left(L_{i}\right)$ and $b_{i}=c_{1}\left(B_{i}\right)$. Then we can write

$$
m=c_{1}(M)=l_{1}+b_{1}=\ldots=l_{n}+b_{n} .
$$

By assumption we have

$$
e^{n, 0}+e^{n-1,1}+\ldots+e^{0, n}=0
$$

in the rational cohomology $H^{2}(X, \mathbb{Q})$, so the classes $e^{p, q}$ can be written in terms of $l_{i}$ in the following way:

$$
e^{n-i, i}=\frac{1}{n+1}\left(-l_{1}-2 l_{2}-\ldots-i l_{i}+(n-i) l_{i+1}+(n-i-1) l_{i+2}+\ldots+l_{n}\right)
$$

for $i=0, \ldots, n$.

Slope $A$-stability of $(E, \theta)$ implies that

$$
\left(e^{0, n}+e^{1, n-1}+\ldots+e^{i, n-i}\right) . A<0
$$

for $i=0, \ldots, n-1$. This is equivalent to

$$
\left(e^{n, 0}+e^{n-1,1}+\ldots+e^{i, n-i}\right) \cdot A>0
$$


for $i=1, \ldots, n$. Rewriting these inequalities in terms of $L_{i}$ we get

$$
N_{i} \cdot A>0
$$

for $i=1, \ldots, n$. Since $L_{i} \leq M$ we have

$$
\sum_{i=1}^{n} x_{i} N_{i} \leq\left(\sum_{i=1}^{n} \frac{i(n-i+1)(n+1)}{2} x_{i}\right) M .
$$

for all non-negative rational numbers $x_{1}, \ldots, x_{n}$. But by Lemma 4.4, $\left.M\right|_{Y}$ is not big and hence the $\mathbb{Q}$-divisor $\sum_{i=1}^{n} x_{i} N_{i}$ restricted to $Y$ is also not big. Therefore we have

$$
\left(\sum_{i=1}^{n} x_{i} N_{i}\right)^{2} \leq 0
$$

for all non-negative rational numbers $x_{1}, \ldots, x_{n}$ (so also for all non-negative real numbers $\left.x_{1}, \ldots, x_{n}\right)$.

This lemma gives many inequalities. Unfortunately, when $n \geq 3$ vanishing of higher Chern classes of $E$ does not seem to give any particularly useful information. For example for $n=3$ in dimension 2 it gives

$$
L_{1}^{2}+\left(L_{1}+2 L_{2}\right)^{2}+\left(L_{1}+2 L_{2}+3 L_{3}\right)^{2}=0
$$

Since

$$
\begin{gathered}
N_{1}=3 L_{1}+2 L_{2}+L_{3}, \\
N_{2}=2 L_{1}+4 L_{2}+2 L_{3}, \\
N_{1}=L_{1}+2 L_{2}+3 L_{3},
\end{gathered}
$$

$E$ is $A$-stable if $L_{2} . A$ is large and $L_{1} . A$ and $L_{3} . A$ are not too negative. In that case $L_{1}^{2}$ can be positive and we do not get an analogue of Proposition 4.3 .

\section{Corollaries}

Recall that our variations of Hodge structure are always considered to be polarizable. We say that a VHS is of weight $k$ if its Hodge decomposition takes the form $V=\bigoplus_{p+q=k, p, q \geq 0} V^{p, q}$. 
Proposition 7.1. Let $X$ be a smooth complex quasi-projective variety. Suppose $\rho_{K}: \pi_{1}(X, x) \rightarrow \mathrm{GL}(n, K)$ is an absolutely irreducible representation defined over an algebraic number field, such that for each embedding $\sigma: K \rightarrow \mathbb{C}$ the associated $\mathbb{C}$-local system $V_{\sigma}$ underlies a polarized complex VHS of weight $k$. Then there is a totally imaginary quadratic extension $L$ of a totally real algebraic number field $F$, with $L$ Galois over $\mathbb{Q}$, and a representation $\rho_{L}: \pi_{1}(X, x) \rightarrow \operatorname{GL}(n, L)$, together with an extension $K^{\prime}$ containing both $K$ and $L$ such that the extensions of scalars of $\rho_{K}$ and $\rho_{L}$ to $K^{\prime}$ are isomorphic (i.e. conjugate). Let $V_{L}$ denote the L-local system corresponding to $\rho_{L}$ and let $V_{L / \mathbb{Q}}$ denote the same local system considered as a local system of $\mathbb{Q}$-vector spaces by restriction of scalars. We may arrange things so that $V_{L / \mathbb{Q}}$ underlies a polarizable $\mathbb{Q}$-variation of Hodge structure of weight $k$.

Proof. This is discussed in [CS, Section 10] and [Si1, Theorem 5]. The basic idea goes back at least to Deligne's "Travaux de Shimura" [Del].

The discussion in the third and following paragraphs of the proof of Theorem 5 in [Si1] does not use the rigidity hypothesis of that theorem, but only the statement that for every embedding $\sigma: K \rightarrow \mathbb{C}$ the induced local system $V_{\sigma}$ is a polarizable VHS. One should assume there that $K$ is a Galois extension of $\mathbb{Q}$ (we can make that hypothesis), and the discussion gives the conclusion that the subfield $L \subset K$ generated by the traces of monodromy elements, has a uniquely defined complex conjugation operation.

We recall the reason. The field of traces that was denoted by $L$ in [Si1] will be denoted by $L^{\text {tr }}$ here, and the extension that we are looking for, denoted $L^{\prime}$ in [Si1], will be denoted by $L$ here.

Since $V_{\sigma}$ is polarizable, its complex conjugate is isomorphic to its dual. As we assume $V$ is absolutely irreducible, each $V_{\sigma}$ is irreducible so this isomorphism is unique up to a scalar.

Let $c: \mathbb{C} \rightarrow \mathbb{C}$ denote complex conjugation. It means that $V_{c \circ \sigma} \cong\left(V_{\sigma}\right)^{*}$. Suppose $\gamma \in \pi_{1}(X, x)$, and let $\rho_{K}^{*}$ denote the dual representation. Recall that $\rho_{K}^{*}(\gamma)=$ $\left(\rho_{K}(\gamma)^{-1}\right)^{t}$, so $\operatorname{Tr}\left(\rho_{K}^{*}(\gamma)\right)=\operatorname{Tr}\left(\rho_{K}\left(\gamma^{-1}\right)\right)$. From the isomorphism $V_{c \circ \sigma} \cong\left(V_{\sigma}\right)^{*}$ we therefore get

$$
(c \circ \sigma)\left(\operatorname{Tr}\left(\rho_{K}(\gamma)\right)\right)=\sigma\left(\operatorname{Tr}\left(\rho_{K}\left(\gamma^{-1}\right)\right)\right) .
$$

As we are assuming that $K$ is a Galois extension of $\mathbb{Q}$, the image of $c \circ \sigma$ is equal to the image of $\sigma$ and it makes sense to write $\sigma^{-1} \circ c \circ \sigma: K \rightarrow K$ (this notational shortcut was used without explanation in [Si1] and required the hypothesis that $K$ be Galois). We obtain

$$
\sigma^{-1} \circ c \circ \sigma\left(\operatorname{Tr}\left(\rho_{K}(\gamma)\right)\right)=\operatorname{Tr}\left(\rho_{K}\left(\gamma^{-1}\right)\right) .
$$

It follows that on the subfield $L^{\text {tr }} \subset K$ generated by the traces of monodromy ele- 
ments, the map

$$
c_{L^{\mathrm{tr}}}:=\sigma^{-1} \circ c \circ \sigma: L^{\mathrm{tr}} \rightarrow L^{\mathrm{tr}}
$$

is well-defined and independent of $\sigma$. In other words, $L^{\text {tr }}$ has a well-defined complex conjugation operation.

Therefore, $L^{\mathrm{tr}}$ is either a totally real field, if $c_{L^{\mathrm{tr}}}$ is the identity, or a totally imaginary quadratic extension of a totally real field $F:=\left(L^{\mathrm{tr}}\right)^{C_{L^{\mathrm{tr}}}}$ otherwise.

Larsen's lemma [Si1, Lemma 4.8] says that we can extend $L^{\text {tr }}$ to $L$ (as said before, that was denoted $L^{\prime}$ there), a totally imaginary quadratic extension of a totally real field, such that $V$ may be defined over $L$. The proof uses a consideration of Brauer groups, and we refer to the discussion in [Si1, Lemma 4.8].

We have

$$
V_{L / \mathbb{Q}} \otimes_{\mathbb{Q}} \mathbb{C}=\bigoplus_{\sigma: L \rightarrow \mathbb{C}} V_{\sigma} .
$$

If $\sigma: L \rightarrow \mathbb{C}$ then it extends to $\sigma_{K^{\prime}}: K^{\prime} \rightarrow \mathbb{C}$ which then restricts to an embedding $\sigma_{K}: K \rightarrow \mathbb{C}$, and $V_{\sigma} \cong V_{\sigma_{K}}$. Therefore, our hypothesis that the $V_{\sigma_{K}}$ are VHS of weight $k$ implies that the $V_{\sigma}$ are VHS of weight $k$.

Our hypothesis says that for any $\sigma$, the Hodge types of $V_{\sigma}$ can be chosen within $\{(p, q): p+q=k$ and $p, q \geq 0\}$. There could be a choice to be made in assigning the Hodge types, for instance if $V_{\sigma}$ is unitary then it has only a single Hodge type and that could be put at any $(p, q)$ in the desired set.

As noted in the discussion of [CS, Section 10.2] we may choose the Hodge types in such a way that there is a $\mathbb{Q}$-polarization. We may do the analogue of [CS, Lemma 10.3] for the general case as follows. Let $c_{L}: L \rightarrow L$ and $c: \mathbb{C} \rightarrow \mathbb{C}$ denote the complex conjugation automorphisms. For $\sigma: L \rightarrow \mathbb{C}$ put $\bar{\sigma}:=c \circ \sigma=\sigma \circ c_{L}$. Choose a collection of embeddings $\sigma_{1}, \ldots, \sigma_{d}: L \rightarrow \mathbb{C}$ such that the $\overline{\sigma_{i}}$ are distinct from the $\sigma_{j}$ and the collection

$$
\left\{\sigma_{1}, \ldots, \sigma_{d}, \bar{\sigma}_{1}, \ldots, \bar{\sigma}_{d}\right\}
$$

is equal to the full collection of embeddings. For each $i$ choose a structure of VHS for $V_{\sigma_{i}}$, and then put

$$
V_{\bar{\sigma}_{i}}^{p, q}:=\overline{V_{\sigma_{i}}^{q, p}} \text {. }
$$

This determines the structures of VHS for $V_{\bar{\sigma}_{i}}$ in such a way that the property of [CS, Lemma 10.3] holds.

As in [CS, Proposition 10.2], there is a form

$$
\Phi: V_{L} \times V_{L} \rightarrow L
$$

which is $\mathbb{Q}$-bilinear, and $L$-linear in the first variable and $L$ - $c_{L}$-antilinear in the second variable that is to say $\Phi(u, \lambda v)=c_{L}(\lambda) \Phi(u, v)$. Also we may assume that 
it is $c_{L^{-}}(-1)^{k}$-symmetric: $\Phi(v, u)=(-1)^{k} c_{L} \Phi(u, v)$ by multiplying by a purely imaginary element if necessary to get this sign right. The form $\Phi$ is unique up to multiplying by an element of $F^{*}$.

Put $\Phi_{\mathbb{Q}}:=\operatorname{Tr}_{L / \mathbb{Q}} \circ \Phi$, giving a $\mathbb{Q}$-bilinear form

$$
\Phi_{\mathbb{Q}}: V_{L / \mathbb{Q}} \times V_{L / \mathbb{Q}} \rightarrow \mathbb{Q}
$$

that is $(-1)^{k}$-symmetric. This will be our polarization form, after having first adjusted $\Phi$ by multiplying by an appropriate element of $F^{*}$ to be chosen below. Let

$$
\Phi_{\mathbb{C}}:\left(V_{L / \mathbb{Q}} \otimes_{\mathbb{Q}} \mathbb{C}\right) \times\left(V_{L / \mathbb{Q}} \otimes_{\mathbb{Q}} \mathbb{C}\right) \rightarrow \mathbb{C}
$$

be the induced $\mathbb{C}$-bilinear form. We would like to say that $\Phi_{\mathbb{C}}$ pairs $V_{\sigma}$ with $V_{\bar{\sigma}}$.

To see this, let $v \mapsto z . v$ be the action of $z \in L$ upon $v \in V_{L / \mathbb{Q}}$. This extends to an action of $L$ on $V_{L / \mathbb{Q}} \otimes_{\mathbb{Q}} \mathbb{C}$ by $\mathbb{C}$-linear automorphisms. Then $V_{\sigma} \subset V_{L / \mathbb{Q}} \otimes_{\mathbb{Q}} \mathbb{C}$ is the subset of vectors such that

$$
z . v=\sigma(z) v
$$

for $z \in L$. We have

$$
\Phi(z . u, v)=\Phi\left(u,\left(c_{L}(z)\right) \cdot v\right) .
$$

Thus, if $u \in V_{\sigma}$ and $v \in V_{\tau}$ then

$$
\begin{aligned}
\Phi_{\mathbb{C}}(z . u, v) & =\Phi_{\mathbb{C}}(\sigma(z) u, v)=\sigma(z) \Phi_{\mathbb{C}}(u, v)=\Phi_{\mathbb{C}}\left(z,\left(c_{L}(z)\right) \cdot v\right) \\
& =\Phi_{\mathbb{C}}\left(u, \tau\left(c_{L}(z)\right) v\right)=\tau\left(c_{L}(z)\right) \Phi_{\mathbb{C}}(u, v)=\bar{\tau}(z) \Phi_{\mathbb{C}}(u, v) .
\end{aligned}
$$

Therefore, if $\sigma(z) \neq \bar{\tau}(z)$ then this expression must be zero. For $\sigma \neq \bar{\tau}$ there is a $z \in L$ with $\sigma(z) \neq \bar{\tau}(z)$ and we obtain $\Phi_{\mathbb{C}}(u, v)=0$. We conclude that $\Phi_{\mathbb{C}}$ pairs $V_{\sigma}$ with $V_{\bar{\sigma}}$.

The complex conjugation on $V_{L / \mathbb{Q}} \otimes_{\mathbb{Q}} \mathbb{C}$ also sends $V_{\sigma}$ to $V_{\bar{\sigma}}$, indeed if $z \cdot v=$ $\sigma(z) v$ then $z \cdot \bar{v}=\overline{z \cdot v}=\overline{\sigma(z) v}=\bar{\sigma}(z) v$.

The Hodge types on $V_{\mathbb{C}}:=V_{L / \mathbb{Q}} \otimes_{\mathbb{Q}} \mathbb{C}$ are defined by

$$
V_{\mathbb{C}}^{p, q}:=\bigoplus_{\sigma} V_{\sigma}^{p, q}
$$

With our choice of Hodge types satisfying the property of [CS, Lemma 10.3], the complex conjugation operation satisfies $\overline{V_{\mathbb{C}}^{p, q}}=V_{\mathbb{C}}^{q, p}$.

We get the hermitian form $(u, v) \mapsto \Phi_{\mathbb{C}}(u, \bar{v})$ on $V_{\sigma}$ induced by $\Phi_{\mathbb{Q}}$. As the hermitian forms on the irreducible local system $V_{\sigma}$ are unique up to multiplication by a real scalar, we deduce that this hermitian form is either a polarization, or minus a polarization of the complex VHS $V_{\sigma}$. By uniqueness, the hermitian form 
has a pure Hodge type, and this type has to be $(k, k)$ otherwise the Hodge types of $V_{\sigma}$ and $V_{\bar{\sigma}}$ could not be symmetric under $(p, q) \mapsto(q, p)$.

In order for $\Phi_{\mathbb{Q}}$ to define a polarization to get a $\mathbb{Q}$-variation of Hodge structure on $V_{L / \mathbb{Q}}$, we need these forms on $V_{\sigma}$ to define polarizations rather than minus polarizations. This problem of fixing the sign, by multiplying our original form $\Phi$ by an element of the totally real field $F^{*}$, was discussed in [CS, Lemma 10.4] and we refer there for the proof, which just uses the fact that $F$ is dense in $F \otimes_{\mathbb{Q}} \mathbb{R}$.

Once this adjustment has been made, we obtain a polarization form $\Phi_{\mathbb{Q}}$, such that with our choice of Hodge decompositions we obtain a $\mathbb{Q}$-variation of Hodge structure $V_{L / \mathbb{Q}}$ of weight $k$.

Remark: In the situation of the proposition, notice that the original irreducible local systems $V_{\sigma_{K}}$ for $\sigma_{K}: K \rightarrow \mathbb{C}$, are among the complex direct factors $V_{\sigma}$ of the $\mathbb{Q}$-variation that was constructed. That was pointed out during the proof.

LEMMA 7.2. Suppose in the situation of the previous proposition, that we know furthermore the traces $\operatorname{Tr}(\rho(\gamma))$ are algebraic integers for $\gamma \in \pi_{1}(X, x)$. Then the $\mathbb{Q}$-VHS constructed there is a $\mathbb{Z}$-VHS.

Proof. By Bass-Serre theory [Ba], there is a projective $\mathscr{O}_{L}$-module $P$ such that $V_{L}=$ $P \otimes_{\mathscr{O}_{L}} L$ (speaking here about the fiber over the basepoint which is a representation of $\pi_{1}(X, x)$ ). Then $P$ gives a local system of free $\mathbb{Z}$-modules forming a lattice inside the $\mathbb{Q}$-local system $V_{L / \mathbb{Q}}$.

Corollary 7.3. Let $X$ be a smooth complex projective variety. Suppose $V$ is a rank 3 local system over an algebraic number field $K$ such that:

1. the monodromy group is Zariski dense in $\mathrm{SL}(3, \bar{K})$;

2. $V$ is integral, in the sense that $\operatorname{Tr}(\rho(\gamma)) \in \mathscr{O}_{K}$ for any group element $\gamma$ (here $\rho$ being the monodromy representation), and

3. for any $\sigma: K \rightarrow \mathbb{C}$ the induced $\mathbb{C}$-local system $V_{\sigma}$ is a VHS.

Then either $V$ projectively factors through an orbicurve, or else the $V_{\sigma}$ are direct factors of the monodromy of a family of abelian varieties.

Proof. Assume that $V$ does not projectively factor through an orbicurve. The same holds for each $V_{\sigma}$. Then by Theorem 5.1, we can conclude that the VHS $V_{\sigma}$ are not of type $(1,1,1)$, because the first case of the theorem is ruled out by our hypothesis that the monodromy group is Zariski dense.

Therefore, the VHS $V_{\sigma}$ may be chosen to have set of Hodge types contained in $\{(1,0),(0,1)\}$. Indeed, if $V_{\sigma}$ is unitary we can just choose one of them; if not, it 
must have Hodge numbers $(1,2)$ or $(2,1)$ since $(1,1,1)$ is ruled out. In either case we can arrange the Hodge types within $\{(1,0),(0,1)\}$. In other words, each $V_{\sigma}$ has a structure of VHS of weight 1.

We may now apply Proposition 7.1 to conclude that the $V_{\sigma}$ is a direct factor in the monodromy of a $\mathbb{Q}$-VHS of weight 1 denoted $V_{L / \mathbb{Q}}$. By Lemma 7.2 using our hypothesis (1), it is a polarized $\mathbb{Z}$-VHS of weight 1 . This corresponds to a family of abelian varieties [Del].

Remark 7.4. Let $V$ be a rank 1 local system defined over an algebraic number field $K$, integral and such that for each embedding $\sigma: K \rightarrow \mathbb{C}$ the associated local system $V_{\sigma}$ is a polarizable VHS. Then $V_{\sigma}$ is unitary so Kronecker's theorem implies that the monodromies of $V$ are roots of unity. In other words $V$ is of finite order (cf. proof of Proposition 2.10).

LEMMA 7.5. If for some $n \geq 1$ there exists a rigid representation $\rho: \pi_{1}(X, x) \rightarrow$ $\mathrm{GL}(n, \mathbb{C})$ then the moduli space of rank 1 local systems is a torsion abelian group. In particular, a rigid local system of rank 1 is of finite order.

Proof. If the moduli space of rank 1 local systems has positive dimension then its connected component of identity is divisible by $n$ and there exists a non-isotrivial family of rank 1 local systems $W$ containing the trivial system and such that $W^{\otimes n}$ is also a non-isotrivial family. If $V_{\rho} \otimes W_{1} \simeq V_{\rho} \otimes W_{2}$ for some rank 1 local systems $W_{1}$ and $W_{2}$ then the determinants coincide and $W_{1}^{\otimes n} \simeq W_{2}^{\otimes n}$. Therefore the family $V_{\rho} \otimes W$ of rank $n$ local systems is non-isotrivial. Since it contains $V_{\rho}$, we get a contradiction with rigidity of $\rho$.

It follows that $H^{1}(X, \mathbb{Q})=0$ and the moduli space of rank 1 local systems is a torsion abelian group, so for any rigid $\rho$ the system $\operatorname{det} V_{\rho}$ is of finite order.

Definition 7.6. Let $\rho: \pi_{1}(X, x) \rightarrow \mathrm{GL}(n, \mathbb{C})$ be a representation. The geometric monodromy group $M_{\text {geom }}(\rho)$ of $\rho$ is the Zariski closure of the image of $\rho$ in $\mathrm{GL}(n, \mathbb{C})$. We say that $\rho$ is properly rigid if $\rho: \pi_{1}(X, x) \rightarrow M_{\text {geom }}(\rho)$ is rigid.

Note that any rigid representation is properly rigid. On the other hand, a properly rigid representation might be nonrigid. For example, it could happen that $H^{1}(X, \mathbb{Q}) \neq 0$. Therefore, it is more general to consider properly rigid representations.

Given a rigid representation into $\operatorname{GL}(n, \mathbb{C})$, by the previous lemma the identity component $M_{\text {geom }}(\rho)^{0}$ of its geometric monodromy group is contained in $\operatorname{SL}(n, \mathbb{C})$. In the following we are interested in properly rigid representations with the largest possible geometric monodromy group. More precisely, we are interested in representations $\rho: \pi_{1}(X, x) \rightarrow \mathrm{GL}(n, \mathbb{C})$ which satisfy one of the following equivalent conditions: 
1. $M_{\text {geom }}(\rho)$ contains $\operatorname{SL}(n, \mathbb{C})$ as a subgroup of finite index,

2. $M_{\text {geom }}(\rho)^{0}=\operatorname{SL}(n, \mathbb{C})$,

3. Lie $M_{\text {geom }}(\rho)=\mathfrak{s l}(n, \mathbb{C})$.

If any of these conditions is satisfied then the composition

$$
\rho: \pi_{1}(X, x) \stackrel{\rho}{\longrightarrow} \mathrm{GL}(n, \mathbb{C}) \stackrel{\operatorname{det}}{\longrightarrow} \mathbb{C}^{*}
$$

maps onto the group of $m$-th roots of unity $\mu_{m}$, where $m$ is the index of $M_{\text {geom }}(\rho)^{0}$ in $M_{\text {geom }}(\rho)$. Then the surjection $\pi_{1}(X, x) \rightarrow \mu_{m}$ gives rise to a degree $m$ étale Galois covering $f: Z \rightarrow X$ such that $M_{\text {geom }}\left(f^{*}(\rho)\right)=\operatorname{SL}(n, \mathbb{C})$.

COROLlary 7.7. Let $V$ be an irreducible complex local system of rank 3. Assume that the corresponding representation is integral, properly rigid and that the geometric monodromy group of $V$ contains $\mathrm{SL}(3, \mathbb{C})$ as a subgroup of finite index. Then either $V$ projectively factors through an orbicurve, or $V$ comes as a direct factor in the monodromy of a family of abelian varieties.

Proof. Rigidity implies that $V$ is defined over an algebraic number field $K$ and it implies that for any $\sigma: K \rightarrow \mathbb{C}$ the induced local system $V_{\sigma}$ is a VHS (see [Si1, Lemma 4.5]). By the above, we can find a finite étale covering $f: Z \rightarrow X$ such that $M_{\text {geom }}\left(f^{*}(\rho)\right)=\operatorname{SL}(n, \mathbb{C})$. Therefore $f^{*}(\rho)$ satisfies Hypothesis (1) of Corollary 7.3. Clearly, $f^{*} V_{\sigma}$ is also a VHS for any $\sigma$ so $f^{*}(\rho)$ satisfies also Hypothesis (3). Hypothesis (2) is provided by our assumption that $V$ is integral. Hence Corollary 7.3 implies that $f^{*} V$ projectively factors through an orbicurve, or else the $f^{*} V_{\sigma}$ are direct factors of the monodromy of a family of abelian varieties.

We may descend these conclusions back to $X$. Lemma 8.6 says that if $f^{*} V$ projectively factors through an orbicurve, then $V$ does so. If on the other hand $f^{*} V$ comes from a family of abelian varieties, then taking the product of the Galois conjugates of the family gives a family which descends back to $X$, and $V$ is a direct factor in the monodromy of this family.

COROLLARY 7.8. If $V$ is any local system of rank 3 for which the geometric monodromy group contains $\mathrm{SL}(3, \mathbb{C})$ as a subgroup of finite index, then either it projectively factors through an orbicurve, or it comes from a family of abelian varieties, or else there is a non-constant equivariant map from the universal cover of $X$ to a two-dimensional building.

Proof. If the representation is non-rigid, or non-integral, then there is a non-constant equivariant map from the universal cover of $X$ to a two-dimensional building (see [GS] and [CS, Section 4]). Otherwise, the previous corollary applies. 
Let us recall that by Lemma 7.5 a rigid local system $V$ has $\operatorname{det}(V)$ of finite order.

COROLlary 7.9. Suppose $V$ is an irreducible rank 3 local system. If $V$ is integral and properly rigid with $\operatorname{det}(V)$ of finite order, then it is of geometric origin.

Proof. Let us first assume that the geometric monodromy group contains $\operatorname{SL}(3, \mathbb{C})$ as a subgroup of finite index. If $V$ projectively factors through an orbicurve, then the factoring representation is rigid. As was shown in [CS] using Katz's theorem, it follows that the factoring representation is of geometric origin. Let us recall briefly the argument here. If an orbicurve admits a rigid representation, then its coarse moduli space is the projective line and there is an open subset of the orbicurve that is isomorphic to $\mathbb{P}^{1}-\left\{t_{1}, \ldots, t_{k}\right\}$. By modifying the monodromy at one point, we may assume that we have an $\operatorname{SL}(3, \mathbb{C})$-local system on the orbicurve, pulling back to a local system projectively equivalent to our original one. It is still rigid as a local system on the orbicurve, which implies also that it is rigid as a local system on $\mathbb{P}^{1}-\left\{t_{1}, \ldots, t_{k}\right\}$ with fixed (semisimple, finite-order) monodromy transformations at the points $t_{i}$. Therefore, Katz's theorem [Ka2] says that the new local system has geometric origin. We may now complete the proof of this case: the last part of Proposition 2.10 shows that our original representation was of geometric origin.

If $V$ does not projectively factor through an orbicurve then Corollary $7.7 \mathrm{im}$ plies that $V$ is a direct factor in the monodromy of a family of abelian varieties, so it is of geometric origin.

If the geometric monodromy group is not a group containing $\operatorname{SL}(3, \mathbb{C})$ then it is either finite, isogenous to $\operatorname{SL}(2, \mathbb{C})$, or isogenous to a positive-dimensional torus. Note however that in the case of a monodromy group isogenous to a positivedimensional torus, the representation is not rigid, indeed a rigid representation to a finite extension of a torus must have finite image. A representation with finite monodromy group has geometric origin, see Proposition 2.10 . For monodromy groups isogenous to $\mathrm{SL}(2, \mathbb{C})$ the result of $[\mathbb{C S}]$ shows that rigid integral local systems are of geometric origin.

Proof of Theorem 1.3. We note that the statement of Theorem 1.3 is a special case of Corollary 7.9. Indeed, assume the hypotheses of Theorem 1.3 hold, then since any rigid representation is properly rigid, and a representation into $\operatorname{SL}(3, \mathbb{C})$ automatically has determinant of finite order, the hypotheses of Corollary 7.9 hold so the local system is of geometric origin.

We leave it to the reader to formulate some other variants on these corollaries. For instance, one could say that a rigid integral representation of rank 3 comes (projectively) by pullback from a curve or a Shimura modular variety. 
The hypothesis of rigidity could be replaced by a hypothesis that the representation is a point of a 0 -dimensional stratum of a stratification by, say, dimensions of cohomology groups or singularities of the moduli space.

We do not know how to show the conjecture "rigid implies integral". One could hope that in some kind of very heuristic sense the argument for the $(1,1,1)$ case could motivate us to analyze what happens when we have a map to a 2-dimensional building.

COROLlARY 7.10. Let $X$ be a smooth projective variety such that for $i=1,2,3$ we have $H^{0}\left(X, \operatorname{Sym}^{i} \Omega_{X}\right)=0$. Then any representation $\pi_{1}(X, x) \rightarrow \operatorname{GL}(3, \mathbb{C})$ is of geometric origin.

Proof. If $X$ is a smooth projective variety such that $H^{0}\left(X, \operatorname{Sym}^{i} \Omega_{X}\right)=0$ for $i=$ $1,2,3$, then any representation $\pi_{1}(X, x) \rightarrow \mathrm{GL}(3, \mathbb{C})$ is rigid and integral (see [K12, Theorem 1.6 and Corollary 1.8]). Corollary 7.9 implies that it is of geometric origin.

Interesting examples of such varieties come from [K12, Theorem 1.11]. Namely we can take the ball quotient $\Gamma \backslash \mathbf{B}_{\mathbb{C}}^{n}$ for a torsion free Kottwitz lattice $\Gamma \subset S U(n, 1)$ for $n \geq 4$ such that $n+1$ is prime. But these varieties are simple Shimura varieties, so probably for such varieties our result follows easily in a different way.

Our results give geometric origin for two-dimensional ball quotients (that is to say, any smooth projective surfaces of general type with $c_{1}^{2}=3 c_{2}$ ), provided we know integrality:

COROLlary 7.11. Suppose $\Gamma \subset P U(2,1)$ is a torsion-free cocompact lattice that is integral, i.e. such that the traces of its elements are algebraic integers. Let $X=B^{2} / \Gamma$ be the ball quotient which is a smooth projective variety. Then the standard representation of $\pi_{1}(X)=\Gamma$ is of geometric origin.

Proof. The standard representation $\rho$ is cohomologically rigid (see below), by [We], so all of its conjugates $\rho^{\sigma}$ for $\sigma \in \operatorname{Gal}(\mathbb{C} / \mathbb{Q})$ are cohomologically rigid, hence they are variations of Hodge structure. There exists a finite possibly ramified covering $Z \rightarrow X$ such that $\rho_{Z}=\left.\rho\right|_{\pi_{1}(Z)}$ lifts to $S U(2,1)$ (Lemma2.6), in other words it is a rank 3 local system. It is clearly Zariski-dense. Furthermore, $\rho_{Z}$ is a variation of Hodge structure where the period map is the composition

$$
\widetilde{Z} \rightarrow \widetilde{X} \cong B^{2},
$$

in particular the differential of this period map at a general point has rank 2. Therefore, $\rho_{Z}$ cannot factor through an orbicurve. Now apply Corollary 7.3 to get that 
$\rho_{Z}$ has geometric origin, and by Proposition 2.10 $\rho$ is of geometric origin (meaning that its composition with any linear representation of $P U(2,1)$ has geometric origin).

Remark 7.12 (Added in revision). A vector bundle $E$ with an integrable connection $\nabla$ on $X / \mathbb{C}$ is called cohomologically rigid if $H^{1}\left(X, \mathscr{E} n d^{0}(E, \nabla)\right)=0$. A local system is cohomologically rigid if the corresponding vector bundle with an integrable connection is cohomologically rigid. Esnault and Groechenig prove that a cohomologically rigid local system is integral [EG]. This means that in many of our statements above we can remove the integrality hypothesis if we assume cohomological rigidity.

An important special case is that this allows us to remove the hypothesis of integrality in Corollary 7.11. Indeed for two-dimensional ball quotients the standard representation $\Gamma \rightarrow P U(2,1)$ is cohomologically rigid by [We], therefore [EG] applies to provide the hypothesis of integrality needed to apply Corollary 7.11 We conclude that the standard representation is of geometric origin, for any smooth projective surface of general type with $c_{1}^{2}=3 c_{2}$.

\section{Appendix. The factorization theorem}

The main aim of this appendix is to prove Theorem 3.3, Before giving proof of this theorem, we need a few auxiliary results. The first one is a strong version of Proposition 2.7 and Remark 2.8 in case of representations into SL $(n, \mathbb{C})$.

Proposition 8.1. Let $X$ be a smooth complex quasi-projective variety with an irreducible representation $\rho: \pi_{1}(X, x) \rightarrow \mathrm{SL}(n, \mathbb{C})$. Suppose $f: X \rightarrow C$ is a fibration (cf. 91.1 ) over an orbicurve such that for a general fiber $F=f^{-1}(c)$, the restriction of $\rho$ to $F$ is reducible. Then one of the following holds:

1. $\rho$ projectively factors through $f$.

2. There exists a finite étale cover $C_{Z} \rightarrow C$, which by base change induces a finite étale cover $p: Z \rightarrow X$ such that $p^{*} \rho$ is tensor decomposable. In particular, $\rho$ is virtually tensor decomposable.

3. There exists a finite étale cover $C_{Z} \rightarrow C$, which induces a finite étale cover $p: Z \rightarrow X$ such that $p^{*} \rho$ is reducible. In particular, $\rho$ is virtually reducible.

Proof. Some arguments in the following proof are similar to the one used, e.g. in [Ka2, p. 92-93]. 
Let $V_{\rho}$ denote the local system associated to $\rho$. Write the isotypical decomposition

$$
\left.V_{\rho}\right|_{F}=\bigoplus V_{i} \otimes W_{i}
$$

where $V_{i}$ are distinct irreducible representations of $\pi_{1}(F, x)$ and $W_{i}$ are vector spaces. By hypothesis, either there is more than one factor, or there is a single factor but $W_{1}$ has dimension $>1$.

Use the homotopy exact sequence (Theorem 2.1), assuming that $C$ has the maximal orbifold structure such that $f$ is a morphism, and letting $x \in F$. Let $\Phi$ denote the image of $\pi_{1}(F, x)$ in $\pi_{1}(X, x)$, which is also the kernel of $\pi_{1}(X, x) \rightarrow \pi_{1}(C, c)$. Then $\pi_{1}(C, c)$ acts by outer automorphisms on $\Phi$ (more precisely, we have a homomorphism from $\pi_{1}(C, c)$ to the group of outer automorphisms of $\left.\Phi\right)$. The above isotypical decomposition is a decomposition of the restriction of $\rho$ to $\Phi$, and inner automorphisms of $\Phi$ preserve the isomorphism type of the $V_{i}$. Therefore, $\pi_{1}(C, c)$ acts on the set of isotypical components $\left\{V_{i}\right\}$ by its conjugation action.

Assume that there is more than one different isotypical component. As there are only finitely many isotypical components, there exists a subgroup of finite index in $\pi_{1}(C, c)$ that stabilizes each isotypical component. Therefore there exists a finite étale covering $p: Z \rightarrow X$ obtained by base change from a finite étale covering $C_{Z} \rightarrow C$ defined by the above subgroup, such that when we pull back the above picture to $Z$, the isotypical components are preserved by the action of $\pi_{1}\left(C_{Z}, c^{\prime}\right)$. Then the decomposition is preserved by the full monodromy action of $\pi_{1}(Z, z)$. So $\left.V_{\rho}\right|_{Z}$ becomes reducible, and in that case $\rho$ is virtually reducible.

We may therefore now suppose that there is only a single isotypical component in the above decomposition, that is to say

$$
\left.V_{\rho}\right|_{F}=V_{1} \otimes W_{1}
$$

where $n_{1}=\operatorname{rk} V_{1} \geq 1$ and $m_{1}=\operatorname{dim} W_{1} \geq 2$.

Lemma 8.2. There exists a finite étale covering $C_{Z} \rightarrow C$ of the orbicurve $C$ such that for the induced étale covering $p: Z \rightarrow X$ inducing $p_{F}: F_{Z} \rightarrow F$, the local system $p_{F}^{*}\left(V_{1}\right)$ extends to a local system $V_{1}^{\prime}$ on $Z$.

Proof. Let $\Phi \subset \pi_{1}(X, x)$ be the image of $\pi_{1}(F, x)$ and let $\varphi: \Phi \rightarrow \operatorname{GL}\left(n_{1}, \mathbb{C}\right)$ be the irreducible representation corresponding to $V_{1}$. The homomorphism $\varphi$ factors through the almost-simple subgroup $G:=\left\{A \in \mathrm{GL}\left(n_{1}, \mathbb{C}\right):(\operatorname{det} A)^{m_{1}}=1\right\}$. For each element $g \in \pi_{1}(X, x)$ we can consider the representation $\varphi_{g}: \Phi \rightarrow G \subset$ $\mathrm{GL}\left(n_{1}, \mathbb{C}\right)$ given by $\varphi_{g}(h)=\varphi\left(g h g^{-1}\right)$ and the corresponding $\Phi$-module $V_{\varphi_{g}}$. By definition, $V_{\varphi} \otimes W \simeq V_{\varphi_{g}} \otimes W$ and $V_{\varphi}$ is a simple $\Phi$-module. Therefore there exists a non-zero map from one of the simple factors in the Jordan-Hölder filtration of $V_{\varphi_{g}}$ 
to $V_{\varphi}$. By Schur's lemma, this factor is isomorphic to $V_{\varphi}$. But $V_{\varphi}$ and $V_{\varphi_{g}}$ have the same dimension over $\mathbb{C}$, so $V_{\varphi_{g}}$ is simple and the $\Phi$-modules $V_{\varphi}$ and $V_{\varphi_{g}}$ are isomorphic. Therefore for each fixed $g \in \pi_{1}(X, x)$, we can choose $A_{g}$ in $G \subset \operatorname{GL}\left(n_{1}, \mathbb{C}\right)$ such that

$$
\varphi\left(g h g^{-1}\right)=A_{g} \varphi(h) A_{g}^{-1}
$$

for all $h \in \Phi$. The map $\bar{\tau}: \pi_{1}(X, x) \rightarrow \operatorname{PGL}\left(n_{1}, \mathbb{C}\right)$ defined by sending $g$ to the class of $A_{g}$, is a projective representation extending the projective representation $\pi_{1}(F, x) \rightarrow \operatorname{PGL}\left(n_{1}, \mathbb{C}\right)$ associated to $V_{1}$.

We distinguish two cases. By [BN, Proposition 5.5] (see also [CS, 2.2]), either the orbicurve is spherical, or it has a finite étale covering that is a usual curve with infinite fundamental group.

In the spherical case, there is a finite étale covering of $C$ whose fundamental group is trivial. After pulling back to this covering we are in the situation where $\Phi=\pi_{1}(X, x)$ and the claim follows.

We may therefore assume that there is a finite étale covering of $C$ that is a usual curve not equal to $\mathbb{P}^{1}$ or $\mathbb{A}^{1}$. Pulling everything back to this covering, we may assume that $C$ has no orbifold structure.

We can choose an analytic neighbourhood $U \subset C$ of $c=f(x)$ isomorphic to a disk and such that the induced fibration $f^{-1}(U) \rightarrow U$ is trivial in the usual topology (cf. [No, Lemma 1.5 A]). If $C$ is compact then we choose a point $c^{\prime} \in U-\{c\}$. The fiber $F^{\prime}$ of $f$ over $c^{\prime}$ is smooth and homeomorphic to $F$. Then we define the Zariski open subcurve $C^{*}=C-\left\{c^{\prime}\right\} \subset C$. In case $C$ is non-complete we set $C^{*}=C$. Let us also set $X^{*}=f^{-1}\left(C^{*}\right)$. By construction, the fundamental group $\pi_{1}\left(C^{*}, c\right)$ is free.

Now for some elements $g_{1}, \ldots g_{s} \in \pi_{1}\left(X^{*}, x\right)$ whose images are free generators of $\pi_{1}\left(C^{*}, c\right)$, we can consider the semidirect product

$$
\Phi \ltimes\left\langle g_{1}, \ldots, g_{s}\right\rangle
$$

with $g_{i}$ acting on $\Phi$ by conjugation. This group is isomorphic to $\pi_{1}\left(X^{*}, x\right)$. Then we can extend $\varphi$ to a representation $\tilde{\varphi}: \pi_{1}\left(X^{*}, x\right) \rightarrow G$ by setting

$$
\tilde{\varphi}\left(h g_{1}^{a_{1}} \ldots g_{s}^{a_{s}}\right)=\varphi(h) A_{g_{1}}^{a_{1}} \ldots A_{g_{s}}^{a_{s}}
$$

The short exact sequence

$$
1 \rightarrow \mu_{l} \rightarrow G \rightarrow \operatorname{PGL}\left(n_{1}, \mathbb{C}\right) \rightarrow 1
$$

where $l=n_{1} m_{1}$, leads to an exact sequence of (non-abelian) group cohomology

$$
H^{1}\left(\pi_{1}(X) ; G\right) \rightarrow H^{1}\left(\pi_{1}(X) ; \operatorname{PGL}\left(n_{1}, \mathbb{C}\right)\right) \stackrel{\delta}{\rightarrow} H^{2}\left(\pi_{1}(X) ; \mu_{l}\right),
$$


where the map $\delta$ sends the 1-cocyle $[\bar{\tau}]=\left\{A_{g}\right\}_{g \in G}$ to the 2-cocycle []: $\pi_{1}(X) \times$ $\pi_{1}(X) \rightarrow \mu_{l}$ given by

$$
[g, h]=A_{g} A_{h} A_{g h}^{-1} .
$$

The sequence above shows that this 2-cocycle is the obstruction to lifting $\bar{\tau}$ to a representation $\pi_{1}(X, x) \rightarrow G$.

Let $\Phi^{*} \subset \pi_{1}\left(X^{*}, x\right)$ be the image of $\pi_{1}(F, x)$. We claim that the canonical surjection $\Phi^{*} \rightarrow \Phi$ is in fact an isomorphism. By van Kampen's theorem $\pi_{1}(X, x)$ is isomorphic to the amalgamated product of $\pi_{1}\left(X^{*}, x\right)$ and $\pi_{1}\left(f^{-1}(U), x\right) \simeq \pi_{1}(F, x)$ over $\pi_{1}\left(X^{*} \cap f^{-1}(U), x\right) \simeq \pi_{1}(F, x) \times \mathbb{Z}$. This implies that $\Phi^{*} \simeq \Phi$.

Therefore we have a commutative diagram

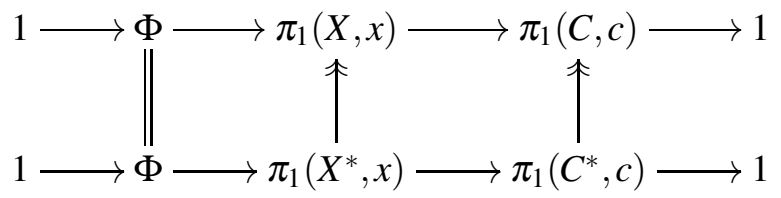

that leads to the commutative diagram

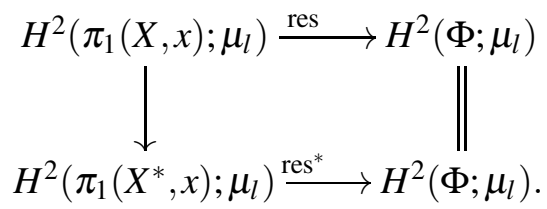

Note that the class $\left.\delta([\bar{\tau}])\right|_{X^{*}} \in H^{2}\left(\pi_{1}\left(X^{*}, x\right) ; \mu_{l}\right)$ is zero, since the representation $\left.\bar{\tau}\right|_{X^{*}}: \pi_{1}\left(X^{*}, x\right) \rightarrow \operatorname{PGL}\left(n_{1}, \mathbb{C}\right)$ lifts to $\tilde{\varphi}: \pi_{1}\left(X^{*}, x\right) \rightarrow G$. Therefore the above diagram shows that $\delta([\bar{\tau}])$ lifts to a class $\eta$ in the kernel of res. Now, by the Lyndon-Hochschild-Serre spectral sequence

$$
H^{p}\left(\pi_{1}(C, c) ; H^{q}\left(\Phi ; \mu_{l}\right)\right) \Rightarrow H^{p+q}\left(\pi_{1}(X, x) ; \mu_{l}\right),
$$

so this kernel fits into the commutative diagram

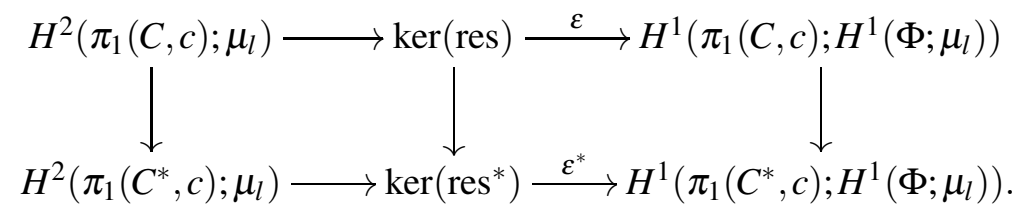

Now we need to kill $\varepsilon(\eta)$ by passing to a finite étale cover of $C$. Let us first remark that $A:=H^{1}\left(\Phi ; \mu_{l}\right)=\operatorname{Hom}\left(\Phi ; \mu_{l}\right)=\operatorname{Hom}\left(\Phi /[\Phi, \Phi] ; \mu_{l}\right)$ is a finite abelian group. This is clear since $\pi_{1}(F, x)$, an hence also $\Phi$, are finitely generated. Passing 
to the finite étale covering defined by the finite index subgroup $\bigcap_{a \in A} \pi_{1}(C, c)_{a} \subset$ $\pi_{1}(C, c)$ obtained by intersecting of all the stabilizers of $\pi_{1}(C, c)$-action on $A$, we can assume that $A$ is a trivial $\pi_{1}(C, c)$-module. Then

$$
H^{1}\left(\pi_{1}(C, c) ; A\right)=\operatorname{Hom}\left(\pi_{1}(C, c), A\right)=\operatorname{Hom}\left(\pi_{1}(C, c) /\left[\pi_{1}(C, c), \pi_{1}(C, c)\right], A\right)
$$

is a finite abelian group. Let us consider a finite index subgroup of $\pi_{1}(C, c)$ defined by

$$
H:=\bigcap_{\varphi \in \operatorname{Hom}\left(\pi_{1}(C, c), A\right)} \operatorname{ker} \varphi \subset \pi_{1}(C, c) .
$$

It is clear from the definition that the induced restriction map $H^{1}\left(\pi_{1}(C, c), A\right) \rightarrow$ $H^{1}(H ; A)$ is zero, so $H \subset \pi_{1}(C, c)$ defines a finite étale cover of $C$ which kills the class $\varepsilon(\eta)$. Therefore we can assume that $\eta$ lifts to a class $\tilde{\eta} \in H^{2}\left(\pi_{1}(C, c) ; \mu_{l}\right)$.

By assumption $C$ is not spherical, so the universal cover $\tilde{C}$ of $C$ is contractible. So the spectral sequence

$$
H^{p}\left(\pi_{1}(C, c) ; H^{q}\left(\tilde{C}, \mu_{l}\right)\right) \Rightarrow H^{p+q}\left(C, \mu_{l}\right)
$$

degenerates to $H^{p}\left(\pi_{1}(C, c) ; \mu_{l}\right)=H^{p}\left(C, \mu_{l}\right)$.

If $C$ is not projective then there is nothing to prove as $H^{2}\left(C, \mu_{l}\right)=0$. So we can assume that $C$ is projective of genus $g \geq 1$. For any finite abelian group $A$ any element $\alpha \in H^{2}(C, A)$ can be killed after passing to a finite étale cover $\pi: C^{\prime} \rightarrow$ $C$. This follows from the fact that $H^{2}(C, A) \simeq A$ and $\pi^{*}: H^{2}(C, A) \rightarrow H^{2}\left(C^{\prime}, A\right)$ is multiplication by the degree of $\pi$ and $\pi_{1}(C, c)$ has a subgroup of index equal to the order of $A$. But $\pi_{1}(C, c)$ contains subgroups of arbitrary finite index, so applying the above remark to the class $\tilde{\eta}$, we can find a connected degree $n$ finite étale covering $C_{Z} \rightarrow C$ such that the pullback of $\tilde{\eta}$ is zero in $H^{2}\left(\pi_{1}\left(C_{Z}, c^{\prime}\right) ; \mu_{l}\right)=$ $H^{2}\left(C_{Z}, \mu_{l}\right)$. So letting $Z$ be the pullback of this covering over $X$ we get that our class in $H^{2}\left(\pi_{1}(X, x) ; \mu_{l}\right)$ pulls back to the zero class in $H^{2}\left(\pi_{1}(Z, z) ; \mu_{l}\right)$. Therefore, when pulled back to $Z$, the representation lifts to $G$.

Let $f_{Z}$ denote the map from $Z$ to $C_{Z}$. Let us consider

$$
\left.V_{1}^{\prime} \otimes \operatorname{Hom}\left(V_{1}^{\prime},\left.V_{\rho}\right|_{Z}\right) \rightarrow V_{\rho}\right|_{Z}
$$

Note that $\left.V_{1}^{\prime}\right|_{F_{Z}}$ is irreducible, since $p_{F}: F_{Z} \rightarrow F$ is an isomorphism (this is why we need $p: Z \rightarrow X$ to be induced from a finite covering of the curves $C_{Z} \rightarrow C$ ). So after restricting the above map to the fiber $F_{Z}$ we get an isomorphism. Since this map is a map of local systems on $Z$, it must be an isomorphism.

If the rank of $V_{1}^{\prime}$ is $\geq 2$ this gives a tensor decomposition over $Z$ so $\rho$ is virtually tensor decomposable. Finally, if $V_{1}^{\prime}$ has rank 1 then $V_{1}$ has rank 1 , so the restriction of $\rho$ to $\pi_{1}(F, x)$ projects to the trivial representation in $\operatorname{PGL}(n, \mathbb{C})$. This means that $\rho$ projectively factors through $f$. This finishes proof of Proposition 8.1 
The proof of the next lemma uses projectivity of the base manifold.

LEMMA 8.3. Let $X$ be a smooth complex projective variety of dimension $\geq 2$. Let $\rho: \pi_{1}(X, x) \rightarrow \operatorname{SL}(n, \mathbb{C})$ be a representation with an infinite image. Assume that $\rho$ virtually projectively factors through an orbicurve. Then there exists a fibration $h: X \rightarrow B$ over a smooth projective curve $B$ such that the restriction of $V_{\rho}$ to a general fibre of $h$ has finite monodromy.

Proof. By assumption there exists an alteration $p: Z \rightarrow X$ for which $p^{*} \rho$ projectively factors through a fibration $f: Z \rightarrow C$ over an orbicurve $C$. Let $Z_{y} \subset Z$ denote the fiber over $y \in C$. Choose $a \in C$ at which the stabilizer group is trivial and the fiber $Z_{a}$ is smooth.

Let us consider $g=(p, f): Z \rightarrow X \times C$. Let $Y \subset X \times C$ be the image of $g$ and let $r_{1}: Y \rightarrow X$ and $r_{2}: Y \rightarrow C$ denote the corresponding projections.

Claim. We have $p\left(Z_{a}\right) \cap p\left(Z_{y}\right)=\emptyset$ for general $y \in C$.

Proof. Assume that $p\left(Z_{a}\right) \cap p\left(Z_{y}\right) \neq \emptyset$ for general $y \in C$. Then $Y \cap\left(p\left(Z_{a}\right) \times C\right)$ contains an irreducible component that dominates $C$. So we can choose a complete irreducible curve $D \subset Z$ such that $g(D)$ is contained in $Y \cap\left(p\left(Z_{a}\right) \times C\right)$ and $f(D)=$ $C$. Let $v: \tilde{D} \rightarrow D \hookrightarrow Z$ be composition of the normalization of $D$ with the canonical inclusion. Then $\tilde{D}$ is a smooth projective curve, $p \circ v$ maps $\tilde{D}$ into $p\left(Z_{a}\right)$ but $f \circ v$ : $\tilde{D} \rightarrow C$ is surjective. The situation can be summed up in the following diagram:

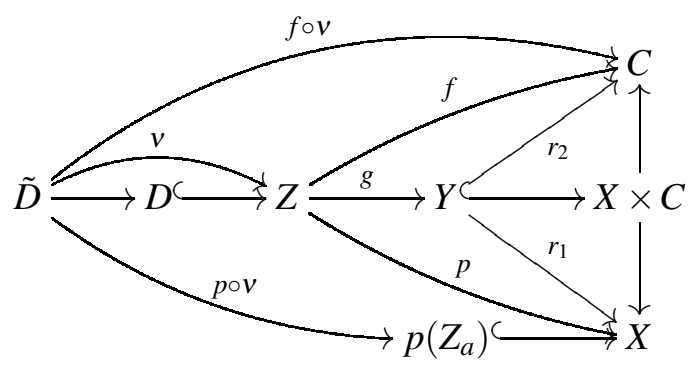

If $p(D)$ is a point then $v^{*} p^{*}\left(V_{\rho}\right)$ is a local system with finite (in fact, trivial) monodromy on $\tilde{D}$. If $D^{\prime}:=p(D)$ is a curve then we can choose an irreducible curve $D^{\prime \prime} \subset Z_{a}$ mapping onto $D^{\prime}$. Since $p^{*} \rho$ projectively factors through $f$, the monodromy of the pullback of $V_{\rho}$ to $Z_{a}$ is contained in the center of $\operatorname{SL}(n, \mathbb{C})$, so it is finite. Therefore the monodromy of the pullback of $V_{\rho}$ to the normalization $\widetilde{D^{\prime \prime}}$ of $D^{\prime \prime}$ is also finite. Since $\widetilde{D^{\prime \prime}} \rightarrow Z \rightarrow X$ factors through the normalization $\widetilde{D^{\prime}}$ of $D^{\prime}$, the image of $\pi_{1}\left(\widetilde{D^{\prime \prime}}\right)$ in $\pi_{1}\left(\widetilde{D^{\prime}}\right)$ has finite index. Therefore the local system $V_{\rho}$, pulled back to the normalization $\widetilde{D}^{\prime}$, has finite monodromy. The map $p \circ v: \tilde{D} \rightarrow X$ factors through $\widetilde{D^{\prime}}$. Therefore, also in this case $v^{*} p^{*}\left(V_{\rho}\right)$ is a local system with finite monodromy on $\tilde{D}$. 
On the other hand, the image of $\pi_{1}(\tilde{D})$ in $\pi_{1}(C)$ has finite index (since $f \circ g$ : $\tilde{D} \rightarrow C$ is surjective), the image of $\rho$ is infinite and $p^{*} \rho$ projectively factors through $f$. Therefore the image of $\pi_{1}(\tilde{D})$ in $S L(n, \mathbb{C})$ cannot be finite, a contradiction.

By the above claim, we can choose three distinct, smooth fibres $Z_{a}, Z_{b}, Z_{c}$ of $f$ such that their images in $X$ are pairwise disjoint divisors. Since $Z_{a}, Z_{b}$ and $Z_{c}$ are algebraically equivalent, the corresponding cycles $p_{*} Z_{a}, p_{*} Z_{b}$ and $p_{*} Z_{c}$ are also algebraically equivalent. In particular, the corresponding cohomology classes of $p\left(Z_{a}\right), p\left(Z_{b}\right)$ and $p\left(Z_{c}\right)$ lie on the same line in $H^{2}(X, \mathbb{Q})$. Therefore by [To1, Theorem 2.1] there exists a fibration $h: X \rightarrow B$ over a smooth curve $B$ such that $p\left(Z_{a}\right), p\left(Z_{b}\right)$ and $p\left(Z_{c}\right)$ are fibers of $h$. Moreover, by [To1, Lemma 2.2] for general $y \in C$, the image $p\left(Z_{y}\right)$ is a fiber of $h$. In particular, $p\left(Z_{y}\right)$ is smooth for general $y \in C$. Since the monodromy group of the pull back of $V_{\rho}$ to $Z_{y}$ is finite and $\pi_{1}\left(Z_{y}\right)$ has finite index in $\pi_{1}\left(p\left(Z_{y}\right)\right)$, we conclude that the restriction of $V_{\rho}$ to a general fiber of $h$ has finite monodromy.

Now we can prove Theorem 3.3 , which we recall:

THEOREM 8.4. Let $X$ be a smooth complex projective variety. Let us fix an irreducible representation $\rho: \pi_{1}(X, x) \rightarrow \operatorname{SL}(n, \mathbb{C})$ for some $n \geq 2$. Suppose that $\rho$ is not virtually tensor decomposable, and not virtually reducible. Then the following conditions are equivalent.

1. $\rho$ projectively factors through an orbicurve;

2. $\rho$ virtually projectively factors through an orbicurve;

3. There exists a nonconstant map $f: X \rightarrow C$ to an orbicurve and a smooth fiber $F=f^{-1}(y)$, such that the restriction of $\rho$ to $\pi_{1}(F, x)$ becomes reducible;

4. There exists an alteration $p: Z \rightarrow X$ such that the previous condition holds for the pullback $p^{*} \rho$.

Proof. Let us first remark that the image of $\rho$ is infinite. Indeed, if the image of $\rho$ is finite then there exists a finite étale covering $X^{\prime} \rightarrow X$ such that the pull back of $\rho$ to $X^{\prime}$ is trivial, contradicting our assumption that $\rho$ is not virtually reducible. In the following we assume that $X$ has dimension $\geq 2$, as otherwise the theorem is trivial. Clearly, the implications (1) $\Rightarrow(2)$ and $(3) \Rightarrow(4)$ are trivial. Let us assume (2). Then by Lemma 8.3 there is a map $h: X \rightarrow B$ to an orbicurve, such that the restriction of $V_{\rho}$ to a general fiber of $h$ has finite monodromy. Apply the homotopy exact sequence (Theorem 2.1), making sure that the orbicurve structure of $B$ is the necessary one so that it works. Let $F$ denote a general fiber. Assume 
that the basepoint $x$ belongs to $F$ and denote by $b:=f(x)$ the basepoint in $B$. We get the exact sequence

$$
\pi_{1}(F, x) \rightarrow \pi_{1}(X, x) \rightarrow \pi_{1}(B, b) \rightarrow 1 .
$$

Now we need the following group-theoretic lemma:

LEMMA 8.5. If $H \subset \mathrm{SL}(n, \mathbb{C})$ is finite and irreducible then the normalizer of $H$ in $\mathrm{SL}(n, \mathbb{C})$ is finite.

Proof. Since $H$ is a closed subgroup of $\operatorname{SL}(n, \mathbb{C})$, its normalizer $G:=N_{H} \operatorname{SL}(n, \mathbb{C})$ is also closed in $\operatorname{SL}(n, \mathbb{C})$. Let us fix an element $h \in H$. Since the identity component $G^{0}$ normalizes $H$, the map $\varphi_{h}: G^{0} \rightarrow \operatorname{SL}(n, \mathbb{C})$ defined by $\varphi_{h}(g)=g h g^{-1} h^{-1}$ has image in $H$. Since $G^{0}$ is connected and $H$ is finite, $\varphi_{h}$ is constant. Hence $G^{0}$ commutes with $H$. Since $H$ is irreducible, elements commuting with $H$ are contained in the centre $Z(\operatorname{SL}(n, \mathbb{C}))$ of $\operatorname{SL}(n, \mathbb{C})$. Therefore $G^{0}$ is finite. Since $G^{0}$ is of finite index in $G, G$ is also finite.

Let $\Phi \subset \pi_{1}(X, x)$ denote the image of $\pi_{1}(F, x)$. Suppose that the restriction $\varphi:=\left.\rho\right|_{\Phi}$ is irreducible and let $H \subset S L(n, \mathbb{C})$ denote the image of $\varphi$. Since $\Phi$ is a normal subgroup of $\pi_{1}(X, x)$, for any $s \in \pi_{1}(X, x), \rho(s)$ is contained in the normalizer of $H$ in $\operatorname{SL}(n, \mathbb{C})$. Therefore, by the above lemma, the image of $\rho$ is finite, contradicting our hypothesis.

Thus the restriction $\left.V_{\rho}\right|_{F}$ to a general fiber is reducible, proving the implication (2) $\Rightarrow(3)$.

Now let us assume (3). Passing to the Stein factorization and using Theorem 2.1, we can assume that $f$ is a fibration over an orbicurve, and the restriction $\left.V_{\rho}\right|_{F}$ to a general fiber of $f$ is reducible. In view of the hypothesis that $\rho$ is not virtually tensor decomposable and not virtually reducible, Proposition 8.1 implies that $\rho$ projectively factors through $f$. This completes the proof of the implication $(3) \Rightarrow$ (1).

The fact that $(3) \Rightarrow(1)$ shows that $(4) \Rightarrow(2)$. Indeed, in the situation of (4), the pullback of $\rho$ to $Z$ satisfies all the hypotheses: it is irreducible because we assumed that $\rho$ was not virtually reducible, and the hypotheses of not being virtually tensor decomposable and not being virtually reducible are conserved. Over $Z$ we are in the situation of (3) so we have shown that $\left.\rho\right|_{Z}$ factors through an orbicurve. This shows $(4) \Rightarrow(2)$ which completes the proof.

In order to optimize the statements of our corollaries in Section 7 we need a result concerning groups lying between $\operatorname{SL}(3, \mathbb{C})$ and $\operatorname{GL}(3, \mathbb{C})$. For notational reasons we do this separately here, rather than modifying the previous discussion 
to take into account such groups. However, one can easily check that the proofs of Proposition 8.1 and Theorem 8.4 work also in this more general case with just minor notational changes.

Suppose $G \subset \mathrm{GL}(3, \mathbb{C})$ is a subgroup such that $\operatorname{SL}(3, \mathbb{C}) \subset G$ is of finite index. Let $\mu_{m}:=G / \mathrm{SL}(3, \mathbb{C})$ denote the quotient group which is a finite cyclic group of some order $m$.

Lemma 8.6. Suppose $\rho: \pi_{1}(X, x) \rightarrow G$ is a representation with Zariski-dense image. Let $Z \rightarrow X$ be the cyclic covering determined by the composed representation $\pi_{1}(X, x) \rightarrow \mu_{m}$, and let

$$
\rho_{Z}: \pi_{1}(Z, z) \rightarrow \mathrm{SL}(3, \mathbb{C})
$$

denote the pullback representation. If $\rho_{Z}$ projectively factors through a map to an orbicurve then $\rho$ projectively factors through a map to an orbicurve.

Proof. Consider the composed representation

$$
\pi_{1}(X, x) \rightarrow G \rightarrow \operatorname{PGL}(3, \mathbb{C}) \stackrel{\text { ad }}{\rightarrow} \operatorname{SL}(8, \mathbb{C})
$$

where the last arrow is the adjoint action on the Lie algebra $\mathfrak{s l}(3) \cong \mathbb{C}^{8}$. Call this representation $\zeta$. Our hypothesis tells us that the monodromy group of $\zeta$ is $\operatorname{PGL}(3, \mathbb{C})$, and that its restriction $\zeta_{Z}$ to $Z$ factors through an orbicurve. As $\operatorname{PGL}(3, \mathbb{C})$ has no finite-index subgroups, the monodromy group of any pullback to a finite cover is still PGL $(3, \mathbb{C})$. In particular, $\zeta$ is not virtually reducible, and not virtually tensor decomposable. By Theorem 8.4 we conclude that $\zeta$ projectively factors through an orbicurve. However, the composed map

$$
\operatorname{PGL}(3, \mathbb{C}) \rightarrow \operatorname{SL}(8, \mathbb{C}) \rightarrow \operatorname{PGL}(8, \mathbb{C})
$$

is injective. Therefore, $\zeta$ factors through an orbicurve. This factorization gives the projective factorization of $\rho$ through an orbicurve, that we are looking for.

\section{Acknowledgements}

The authors would like to thank the referees for their remarks.

The first author was partially supported by Polish National Centre (NCN) contract number 2015/17/B/ST1/02634. This collaboration started during the first author's visit to Université de Nice - Sophia Antipolis in May-June 2015. The visit was supported by Szolem Mandelbrojt prize awarded to the first author by French Mathematical Society (SMF) and Institute Français in Poland.

The second author was partially supported by French ANR grants TOFIGROU (ANR-13-PDOC-0015) and Hodgefun (ANR-16-CE40-0011). 


\section{References}

[Ba] Bass, Hyman, Groups of integral representation type. Pacific J. Math. 86 (1980), 15-51.

[Be] Beauville, Arnaud, Annulation du $H^{1}$ pour les fibrés en droites plats. Complex algebraic varieties (Bayreuth, 1990), 1-15, Lecture Notes in Math., 1507, Springer, Berlin, 1992.

[BN] Behrend, Kai; Noohi, Behrang, Uniformization of Deligne-Mumford curves. J. Reine Angew. Math. 599 (2006), 111-153.

[Bh] Bhatt, Bhargav, Annihilating the cohomology of group schemes. Algebra Number Theory 6 (2012), 1561-1577.

[Bo] Bogomolov, Fedor Alekseivich, Holomorphic tensors and vector bundles on projective manifolds. Izv. Akad. Nauk SSSR Ser. Mat. 42 (1978), 1227-1287, 1439.

[Bor] Borel, Armand, Linear algebraic groups. Second edition. Graduate Texts in Mathematics, 126. Springer-Verlag, New York, 1991. xii+288 pp.

[CD] Catanese, Fabrizio; Dettweiler, Michael, Answer to a question by Fujita on variation of Hodge structures, in Higher Dimensional Algebraic Geometry: In Honor of Professor Yujiro Kawamata's 60th Birthday, Adv. Stud. Pure Math., 74. 2017, 73-102.

[CS] Corlette, Kevin; Simpson, Carlos. On the classification of rank-two representations of quasiprojective fundamental groups. Compos. Math. 144 (2008), 1271-1331.

[Co] Cousin, Gaël, Projective representations of fundamental groups of quasiprojective varieties: a realization and a lifting result. C. R. Math. Acad. Sci. Paris 353 (2015), 155-159.

[De] Debarre, Olivier, Complex tori and abelian varieties. SMF/AMS Texts and Monographs 11. American Mathematical Society, Providence, RI; Société Mathématique de France, Paris, 2005. x+109 pp.

[Del] Deligne, Pierre, Travaux de Shimura. Séminaire N. Bourbaki (1970/71), exp. 389 Springer Lecture Notes in Math., 244 (1971), 123-165.

[dJ] de Jong, Aise Johan, A conjecture on arithmetic fundamental groups. Israel J. Math. 121 (2001), 61-84. 
[EG] Esnault, Hélène; Groechenig, Michael, Rigid connections, F-isocrystals and integrality, preprint, arXiv:1707.00752.

[EV] Esnault, Hélène; Viehweg, Eckart, Lectures on vanishing theorems. DMV Seminar 20. Birkhäuser Verlag, Basel, 1992. vi+164 pp.

[Fu] Fujita, Takao, The sheaf of relative canonical forms of a Kähler fiber space over a curve, Proc. Japan Acad., Ser. A 54 (1978), 183-184.

[GL] Green, Mark; Lazarsfeld, Robert, Higher obstructions to deforming cohomology groups of line bundles. J. Amer. Math. Soc. 4 (1991), 87-103.

[GS] Gromov, Mikhail; Schoen, Richard, Harmonic maps into singular spaces and $p$-adic superrigidity for lattices in groups of rank one. Inst. Hautes Études Sci. Publ. Math. No. 76 (1992), 165-246.

[HL] Hamm, Helmut A.; Lê Dũng Tráng, Lefschetz theorems on quasiprojective varieties. Bull. Soc. Math. France 113 (1985), 123-142.

[IS] Iyer, Jaya N. N.; Simpson, Carlos T., A relation between the parabolic Chern characters of the de Rham bundles. Math. Ann. 338 (2007), 347-383.

[Ka1] Katz, Nicholas M., Rigid local systems. Annals of Mathematics Studies, 139. Princeton University Press, Princeton, NJ, 1996. viii+223 pp.

[Ka2] Katz, Nicholas M., L-functions and monodromy: four lectures on Weil II. Adv. Math. 160 (2001), 81-132.

[K11] Klingler, Bruno, Sur la rigidité de certains groupes fondamentaux, l'arithméticité des réseaux hyperboliques complexes, et les "faux plans projectifs". Invent. Math. 153 (2003), 105-143.

[K12] Klingler, Bruno, Symmetric differentials, Kähler groups and ball quotients. Invent. Math. 192 (2013), 257-286.

[La1] Langer, Adrian, A note on Bogomolov's instability and Higgs sheaves, in Algebraic Geometry: A Volume in Memory of Paolo Francia, (eds. M. Beltrametti et al.), Walter de Gruyter, 2002, 237-256.

[La2] Langer, Adrian, Bogomolov's inequality for Higgs sheaves in positive characteristic. Invent. Math. 199 (2015), 889-920.

[La3] Langer, Adrian, The Bogomolov-Miyaoka-Yau inequality for logarithmic surfaces in positive characteristic. Duke Math. J. 165 (2016), 2737-2769, 
[Laz] Lazarsfeld, Robert, Positivity in algebraic geometry. I. Classical setting: line bundles and linear series. Ergebnisse der Mathematik und ihrer Grenzgebiete. 3. Folge. A Series of Modern Surveys in Mathematics, 48. SpringerVerlag, Berlin, 2004. xviii+387 pp.

[Mo1] Mochizuki, Takuro, Kobayashi-Hitchin correspondence for tame harmonic bundles and an application. Astérisque No. 309 (2006), viii+117 pp.

[Mo2] Mochizuki, Takuro, Kobayashi-Hitchin correspondence for tame harmonic bundles. II. Geom. Topol. 13 (2009), 359-455.

[No] Nori, Madhav V., Zariski's conjecture and related problems. Ann. Sci. École Norm. Sup. (4) 16 (1983), 305-344.

[Si1] Simpson. Carlos T., Higgs bundles and local systems. Inst. Hautes Études Sci. Publ. Math. 75 (1992), 5-95.

[Si2] Simpson, Carlos T., Lefschetz theorems for the integral leaves of a holomorphic one-form. Compositio Math. 87 (1993), 99-113.

[Si3] Simpson, Carlos T., Subspaces of moduli spaces of rank one local systems. Ann. Sci. École Norm. Sup. (4) 26 (1993), 361-401.

[To1] Totaro, Burt, The topology of smooth divisors and the arithmetic of abelian varieties. Michigan Math. J. 48 (2000), 611-624.

[To2] Totaro, Burt, Euler and algebraic geometry. Bull. Amer. Math. Soc. 44 (2007), 541-559.

[We] Weil, André, Discrete subgroups of Lie groups, II. Annals of Math. 75 (1962), 97-123

[Xi] Xiao, Gang, $\pi_{1}$ of elliptic and hyperelliptic surfaces. Internat. J. Math. 2 (1991), 599-615.

[Zu] Zuo, Kang, Representations of fundamental groups of algebraic varieties. Lecture Notes in Mathematics, 1708. Springer-Verlag, Berlin, 1999. viii+135 pp. 